\title{
Diffusion from Convection
}

\author{
M. Medenjak ${ }^{*}$, J. De Nardis ${ }^{2}$, T. Yoshimura ${ }^{3,4}$
}

1 Institut de Physique Théorique Philippe Meyer, École Normale Supérieure,

PSL University, Sorbonne Universités, CNRS, 75005 Paris, France

2 Department of Physics and Astronomy, University of Ghent, Krijgslaan 281, 9000 Gent, Belgium.Department of Physics and Astronomy, University of Ghent, Krijgslaan 281, 9000

Gent, Belgium.

3 Institut de Physique Théorique Philippe Meyer, École Normale Supérieure,

PSL University, Sorbonne Universités, CNRS, 75005 Paris, France

4 Department of Mathematics, King's College London, Strand, London WC2R 2LS, U.K.

* medenjak@lpt.ens.fr

July 9,2020

\begin{abstract}
We introduce non-trivial contributions to diffusion constants in generic manybody systems with Hamiltonian dynamics arising from quadratic fluctuations of ballistically propagating, i.e. convective, modes. Our result is obtained by expanding the current operator in terms of powers of local and quasi-local conserved quantities. We show that only the second-order terms in this expansion carry a finite contribution to diffusive spreading. Our formalism implies that whenever there are at least two coupled modes with degenerate group velocities the system behaves super-diffusively, in accordance with non-linear fluctuating hydrodynamics. Finally, we show that our expression saturates the exact diffusion constants in quantum and classical interacting integrable systems, providing a general framework to derive these expressions.
\end{abstract}

\section{Contents}

1 Introduction 2

2 Diffusion in Hamiltonian many-body systems 3

3 Hydrodynamics on the super-lattice 4

4 Drude weights 5

5 Diffusion constants 6

6 Lower bounds on diffusion $\quad 7$

7 Diffusion in integrable systems $\quad 7$ 
8 NLFHD and super-diffusion: 8

9 Conclusion 8

S1 Extra details on derivation of hydrodynamics from the lattice 9

S1.1 Correlations on super-lattice 9

S1.2 Consistency condition 12

S2 Extra Derivations of equations 13

S2.1 Derivation of Drude weights 13

S2.2 Derivation of the Onsager matrix 14

S2.3 Derivation of the $G$-tensor and diffusion in integrable systems 16

S3 Quadratic lower bound in the normal mode basis 19

S4 Remaining terms in operator expansion $\quad 20$

References $\quad 21$

\section{Introduction}

From its inception statistical physics has strived to derive the laws of hydrodynamics and thermodynamics. Its strength lies in the universality of the results, where only few aspects of microscopic system can influence the physics on macroscopic level. One of the outstanding open problems in the field is to explain the transport behavior of strongly interacting many-body systems by identifying the relevant degrees of freedom regulating the dynamics on hydrodynamical scales. While the emergence of ideal transport in interacting systems has been connected to the presence of local conservation laws, which prevent the decay of the current [1-3], much less is known about the microscopic origins of diffusive transport in Hamiltonian systems. Proving the emergence of diffusive transport and in particular computing diffusion constants in generic interacting many-body systems directly from their Hamiltonian reversible dynamics is still largely an open question [4]. This is a very non-trivial task even with the powerful numerical methods available for one-dimensional systems [5 7]. Recently, many non-trivial analytical results were obtained in holographic matter [8 12], random or noisy models $\left[\begin{array}{ll}13 & 16\end{array}\right.$ and calculated numerically in some generic chaotic systems $[6]$. The first analytical results on diffusion in reversible many-body classical systems date back to the second half of previous century and the studies of hard rod gasses [17-19]. The research has been reinvigorated in past years by the advances in the theory of quantum integrable systems 20 25], following the surprisingly discovery that in the presence of interactions the diffusive spreading occurs despite integrability.

Regardless of these developments, a clear account of the mechanism leading to diffusion in integrable systems, that could provide the connection with the transport properties of generic systems, is still missing. In this article we fill this void by deriving a closed-form expression for the contribution to diffusion coefficients arising from the interaction of convective modes. 
While we require that the system exhibits convection, i.e. ballistic, transport for some degrees of freedom we make no assumptions about the integrability structure, which makes our theory applicable to general many-body systems with at least one conserved quantity [26, for instance quantum fluids with translational invariance symmetry [27, 28], non-integrable anharmonic chains [29, and non-integrable cellular automata 21,30]. Surprisingly, the convective contribution to diffusion relies only on stationary properties of conservation laws and their currents.

The diffusion constant is obtained by the power series expansion of the current operator within the hydrodynamical cell in terms of conservation laws. A part of diffusion constant can then be related to the projection of the current onto the second-order term in this expansion. This contribution arises as a consequence of the dispersion of convective modes in thermal or non-thermal ensembles. On the level of the second-order term in this expansion, the Euler scale equations for the eigenmodes of the system can be used to derive a closed-form expression which saturates the diffusion constants in integrable theories [22,23,31]. A natural connection can also be made with a lower bound on the diffusion constant in terms of quadratically extensive quantities [32], and in terms of the curvature of Drude weights [33]. It should be stressed that we do not assume the presence of randomness in the dynamics

The approach is reminiscent of the non-linear fluctuating hydrodynamics (NLFHD) theory [28, 29, 34, 35], which employs the expansion of the currents in terms of conservation laws. Importantly, however, NLFHD is a phenomenological theory which cannot, at the moment, be used to evaluate the diffusion constant. The main accomplishment of NLFHD was to show that the presence of a quadratic coupling of the modes in the second-order expansion gives rise to Kardar-Parisi-Zhang [36] or Lévy super-diffusive universal transport. Generalization of this result manifests itself within the framework of our theory as a divergence of the diffusion constant in the presence of degenerate group velocities.

\section{Diffusion in Hamiltonian many-body systems}

Let us consider the Hamiltonian dynamics on an infinite chain $k \in \mathbb{Z}$. The conservation of energy implies continuity equation for energy density $h_{k}$,

$$
\partial_{t} h_{k}(t)-j_{k+1}(t)+j_{k}(t)=0 \text {. }
$$

For $t=0$ or $k=0$ the corresponding space/time coordinate will be omitted. Diffusion constant describes how a localized energy packet spreads through the system in thermal equilibrium, and can be therefore defined in terms of the variance of dynamical structure factor 31.

$$
\frac{1}{C} \sum_{y} y^{2}\left\langle h_{y}(t), h\right\rangle=\frac{D}{C} t^{2}+\mathfrak{D} t+\mathcal{O}(1),
$$

where $C$ is susceptibility, $D$ Drude weight, and $\mathfrak{D}$ the diffusion constant. Here we introduced a connected correlation function

$$
\langle a, b\rangle=\langle a b\rangle-\langle a\rangle\langle b\rangle
$$

with respect to the thermal average $\langle\bullet\rangle=\frac{\operatorname{tr}(\bullet \rho(\beta))}{\operatorname{tr}(\rho(\beta))}, \quad \rho(\beta)=\exp (-\beta H)$. Using the continuity equation and $\mathcal{P} \mathcal{T}$ invariance of Hamiltonian dynamics one can relate the diffusion constant to 


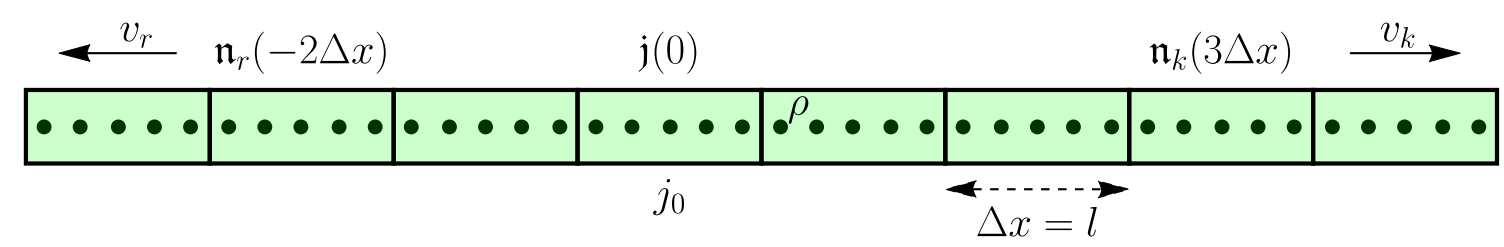

Figure 1: Schematic representation of the contribution to diffusion from convective modes. Current operator at origin excite two normal modes on top of the density matrix $\rho$, which traverse the system with fixed velocities. The normal modes give the contribution to the Onsager matrix when they reach the current operator at position $\chi$.

the Onsager matrix $\mathfrak{L}=\mathfrak{D} C$, where the latter corresponds to the current-current correlation function 31

$$
\mathfrak{L}=\sum_{x} \int \mathrm{d} t\left(\left\langle j_{x}(t), j\right\rangle-D\right) .
$$

Importantly, the Onsager matrix is connected to the response of the current to the linear gradient of the external field in the Kubo formalism [37.

The above discussion can be trivially generalized to the systems with multiple conservation laws $\{q\}$ by introducing Drude weight, susceptibility, diffusion constant and Onsager matrices.

\section{$3 \quad$ Hydrodynamics on the super-lattice}

The goal of hydrodynamics is to identify relevant degrees of freedom which survive on large space-times scales, and thus naturally effect the diffusion constant and Drude weight (2). In order to identify hydrodynamic degrees of freedom the lattice is decomposed into the fluid cells of length $\Delta x=\ell$, and hydrodynamic densities are obtained as sums of (quasi)local operators $o_{k}$ within the fluid cell, and represented by the fraktur font $\mathfrak{o}(\chi, t)=\sum_{k=x-\ell / 2+1}^{x+\ell / 2} o_{k}(t)$, where $\chi=x / \ell$ is the rescaled coordinate. The operators which extend throughout the chain are denoted by capital letters $O(t)=\sum_{\chi} \mathfrak{o}(\chi, t)$

The main hydrodynamic assumption asserts that in the large time limit many-body systems locally equilibrate [38], and that the complete information about the dynamics is contained in local conserved quantities $Q_{i}=\sum_{\chi} \mathfrak{q}_{i}(\chi)$, with $i=1, \ldots, n_{c}$. This leads us to introduce the set of locally equilibrated maximum entropy ensembles

$$
\rho(\underline{\beta}(\chi))=\exp \left(\beta^{i}(\chi) \mathfrak{q}_{i}(\chi)\right),
$$

with temperatures $\underline{\beta}=\left(\beta^{1}, \beta^{2}, \cdots\right)$ and Einstein's repeated indices summation convention $a^{i}(\chi) \mathfrak{o}_{i}(\chi)=\sum_{i=1}^{N} \sum_{\chi} a^{i}(\chi) \mathfrak{o}_{i}(\chi)$. The average, $\langle\bullet\rangle=\frac{\operatorname{tr}(\bullet \rho(\underline{\beta}(\chi)))}{\operatorname{tr}(\rho(\underline{\beta}(\chi)))}$, is always taken with respect to some homogeneous density matrix $\beta^{k}(\chi)=\beta^{k}$, except if expression involves derivatives with respect to temperatures $\beta^{k}(\chi)$, or expectation value of the charge $\mathfrak{q}_{i}(\chi)=\left\langle\mathfrak{q}_{i}(\chi)\right\rangle$, in which case, the homogeneous limit is taken after derivatives.

Since the transport coefficients are related to the asymptotic behavior of two point functions we will consider the following scaling limit $x=\chi \times \ell$ and $t=\tau \times \ell$ with $\ell \rightarrow \infty$. The hydrodynamical assumption asserts that in the scaling limit any operator $\mathfrak{o}(\chi)$ can be 
expressed as a function of conserved charges $\mathfrak{o}(\chi)=f(\underline{\mathfrak{q}})$ and their powers, which is related to local equilibration. There have recently been many works showing that in the homogeneous setup equilibration to the state depending only on conservation laws occurs 39 . Up to the second order the expansion in terms of local charges takes the form

$$
\delta \mathfrak{o}(\chi)=\left(\partial_{\mathbf{q}_{i}(\chi)}\langle\delta \mathfrak{o}(\chi)\rangle\right) \delta \mathfrak{q}_{i}(\chi)+\frac{1}{2}\left(\partial_{\mathbf{q}_{j}(\chi)} \partial_{\mathbf{q}_{i}(\chi)}\langle\delta \mathfrak{o}(\chi)\rangle\right) \delta \mathfrak{q}_{i}(\chi) \delta \mathfrak{q}_{j}(\chi)+\mathcal{R}
$$

with $\delta \mathfrak{o}=\mathfrak{o}-\langle\mathfrak{o}\rangle$, where $\delta \mathfrak{q}_{i_{k}}$ correspond to the densities of (quasi)local conservation laws. The expansion (6) admits an immediate physical interpretation: it corresponds to the variation of the stationary expectation value of observable with respect to the expectation values of conserved quantities. Simply put, on the hydrodynamical scale the perturbation of local equilibrium by an operator $\mathfrak{o}$ can be obtained by projecting the operator on corresponding charges 40. In particular, we can verify that such an expansion satisfies a consistency condition on the level of two point functions $\left\langle\delta \mathfrak{o}_{1}, \delta \mathfrak{o}_{2}\right\rangle$ obtained by expanding only $\delta \mathfrak{o}_{1}$ or both operators $\delta \mathfrak{o}_{1}$ and $\delta \mathfrak{o}_{2}$ S1.2. The remainder terms $\mathcal{R}$ can include non-local charges, higher order contributions, and contributions from charges within neighbouring cells as outlined in S4. In what follows we will focus solely on the contribution to diffusion constant arising from the second order of our expansion, i.e. the convective modes.

In order to determine the contribution to diffusion constant from expansion (6), we have to deduce the dynamics of the second order term in the leading order in $\ell$, i.e. Euler scale. This is easily obtained by solving the continuity equation $\partial_{\tau} \mathfrak{q}(\chi, t)+(\mathfrak{j}(\chi+1, \tau)-\mathfrak{j}(\chi, \tau))=0$, using only the first order contribution from the expansion of the current $\mathfrak{j}$. The solution of the linear differential equations can be expressed in terms of normal modes $\mathfrak{n}_{i}$, which are orthonormal linear combinations of charges $\mathfrak{q}_{i}=\left(R^{-1}\right)_{i}{ }^{j} \mathfrak{n}_{j}[35$. Orthonormality condition implies that $\left(R C R^{\mathrm{T}}\right)_{i j}=\delta_{i j}$, where $C_{i j}=\frac{1}{\ell}\left\langle\mathfrak{q}_{i}(0), \mathfrak{q}_{j}(0)\right\rangle$ is the susceptibility matrix. Physically, normal modes correspond to the localized wave-packets on top of the density matrix $\rho(\underline{\beta})$ which move with distinct velocities $v_{k}$. This means that in the Fourier space $\hat{n}_{i}(k, \tau)=\sum_{\chi} e^{-\mathrm{i} k \chi} n(\chi, \tau)$ they satisfy the continuity equation

$$
\partial_{\tau} \hat{n}_{i}(k, \tau)+\mathrm{i} \omega_{i}(k) \hat{n}_{i}(k, \tau)=0,
$$

with $\omega_{i}(k)=v_{i} k$. Equation (7) admits the corrections of the order $\mathcal{O}\left(\ell^{-1}\right)$, due to the spreading and diffusion of wave-packets, which we here disregard as we are interested in the convective contribution to diffusion constant (see also [40]).

\section{Drude weights}

To demonstrate the utility of our expansion (6), we employ it to obtain the Drude weights, namely the coefficients parametrising the ballistic direct conductivities [41, 42, which are defined as $D_{k l}=\lim _{t \rightarrow \infty}(2 t)^{-1} \int_{-t}^{t} \mathrm{~d} s \sum_{x}\left\langle j_{k, 0}(s), j_{l, x}(0)\right\rangle$, for some mode $k$ and $l$. In terms of the hydrodynamical currents the matrix of Drude weights reads

$$
D_{k l}=\lim _{\tau \rightarrow \infty} \frac{1}{2 \tau \ell} \sum_{\chi} \int_{-\tau}^{\tau} \mathrm{d} \tau^{\prime}\left\langle\mathfrak{j}_{k}\left(0, \tau^{\prime}\right), \mathfrak{j}_{l}(\chi, 0)\right\rangle .
$$


The only contribution to the Drude weight (8) that remains finite after the hydrodynamical expansion (6) in the limit $\ell \rightarrow \infty$ corresponds to the linear order, which reproduces a wellknown result $19,43 ., D_{k l}=\left(\partial_{\mathrm{q}_{i}(0)}\left\langle\mathfrak{j}_{k}(0)\right\rangle\right)\left\langle Q_{i}, j_{l, 0}\right\rangle=B C^{-1} B$, with $B_{i k}=\left\langle\mathfrak{q}_{i}(0), \mathfrak{j}_{k}(0)\right\rangle_{n}$. In the normal mode basis the Drude weights read

$$
D_{k l}=\left\langle j_{k}, N^{k}\right\rangle\left\langle N_{k}, j_{l}\right\rangle
$$

where we introduced extensive normal modes $N_{i}=R_{i}^{j} Q_{j}$. The physical interpretation of this result is the following, see also Fig. 1. The current operator at the origin $\mathfrak{j}_{l}(0)$ creates excitations on top of the density matrix $\rho(\beta)$. The only stable excitations, i.e. excitations which survive the hydrodynamical limit $\ell \rightarrow \infty$, and which are able to reach the current operator at point $\chi, \mathfrak{j}_{l}(\chi)$, are the densities of conserved charges. In the normal mode basis excitation has a well defined velocity $v_{k}$ and gives a contribution to the Drude weight when it reaches the current operator $\mathfrak{j}_{l}(\chi)$.

\section{Diffusion constants}

As already explained the Onsager coefficients are related to the diffusion constant via Einstein's relation $\mathfrak{L}_{k l}=\mathfrak{D}_{k}^{j} C_{j l} 31,44$. We can represent it compactly in terms of the sub-ballistic current $\mathfrak{j}_{k}^{-}(\chi, \tau)=\mathfrak{j}_{k}(\chi, \tau)-\left(\partial_{\mathbf{q}_{i}(\chi)}\left\langle\mathfrak{j}_{k}(\chi)\right\rangle\right) \mathfrak{q}_{i}(\chi, \tau)$, as

$$
\mathfrak{L}_{k l}=\sum_{\chi} \int \mathrm{d} \tau\left\langle\mathfrak{j}_{k}^{-}(\chi, \tau), \mathfrak{j}_{l}^{-}(0,0)\right\rangle .
$$

Plugging the expansion for the current (6), and the Euler scale dynamics of charges (7) produces a finite convective contribution $\mathfrak{L}_{k l}^{c}$ to the Onsager matrix, following a lengthy but elementary manipulation (see $\mathrm{S} 2.2$ for details)

$$
\mathfrak{L}_{k l}^{c}=2\left(R^{-1} \tilde{G}^{2} R^{-\mathrm{T}}\right)_{k l},
$$

where we introduced the renormalized coupling coefficient

$$
\tilde{G}_{i j}^{2}=\frac{G_{i i^{\prime} j^{\prime}} G_{j}^{i^{\prime} j^{\prime}}}{\left|v_{i^{\prime}}-v_{j^{\prime}}\right|},
$$

expressed in terms of the quadratic matrix $G_{i}^{j k}=R_{i}^{l}\left(\left(R^{-1}\right)^{\mathrm{T}} H_{l} R^{-1}\right)^{j k} / 2$ and Hessian $H_{v}^{i j}=\ell \partial_{\mathbf{q}_{j}(0,0)} \partial_{\mathrm{q}_{i}(0,0)}\left\langle\mathbf{j}_{v}(0,0)\right\rangle$.

Once again the result admits a simple physical interpretations (reminiscent of a kineticlike picture [23]). At time $\tau$ the nonzero contribution is produced by excitations created by the currents $\mathfrak{j}(\chi, \tau)$, that reach the operator at the origin $\mathfrak{j}(0,0)$. The weight of contribution is given by the overlap of two normal modes and the current, see FIG 1 . To be more quantitative the Hessian $H_{v}^{i j}$, is rotated by $R$ 's when changing the basis from charge densities $\mathfrak{q}_{i}$ to normal modes $\mathfrak{n}_{i}$, while the renormalization $\left|v_{i^{\prime}}-v_{j^{\prime}}\right|$ arises from the scattering of two normal modes $n_{i^{\prime}}$ and $n_{j^{\prime}}$ with current: $\int \mathrm{d} \tau e^{-\mathrm{i}\left(\omega_{i^{\prime}}(k)+\omega_{j^{\prime}}(-k)\right) \tau}=\frac{2 \pi \delta(k)}{\left|v_{i^{\prime}}-v_{j^{\prime}}\right|}$. The convective Onsager matrix can again be nicely represented in a closed form in terms of normal modes (see $\mathrm{S} 2.2$ and also [40])

$$
\mathfrak{L}_{k l}^{c}=\frac{\left\langle j_{k}^{-}, N_{i} N_{j}\right\rangle\left\langle N^{i} N^{j}, j_{l}^{-}\right\rangle}{2\left|v_{i}-v_{j}\right|} .
$$


In what follows we will check the validity and new implications of our result (13), by comparing its predictions with previous results.

\section{$6 \quad$ Lower bounds on diffusion}

The diagonal elements of Onsager matrix $\mathfrak{L}_{k k}^{c}$ are generally expected to be a lower bound for the exact ones. There have been several proposals in the past years to lower bound the diffusion coefficients. Here we establish a direct connection between our expression (11) and two recent results.

The first bound on diagonal diffusion coefficients $\mathfrak{L}_{k, k}^{s} \geq\left(\left\langle J_{k}, Q\right\rangle\right)^{2} /\left(8 v_{\mathrm{LR}}\langle Q, Q\rangle\right)$ at infinite temperature $\langle\bullet\rangle=\frac{\operatorname{tr}(\bullet)}{\operatorname{tr}(\mathbb{1})}$ was derived in 32 . Here $v_{\mathrm{LR}}$ is the Lieb-Robinson velocity and $Q$ is a conserved quantity which scales quadratically $\langle Q, Q\rangle \sim L^{2}$ with the system size $L$. A set of such conservation laws can be obtained by multiplying two local integrals of motion $Q=\sum_{i \geq j} \alpha_{i j} N_{i} N_{j}$, where we choose traceless $N_{i}$. Provided that all of the quadratically extensive quantities are of this form, we can show that our expression supersedes this lower bound for arbitrary values of coefficients $\alpha_{i j}$ (see $\mathrm{S} 3$ for the details). However, additional quadratically extensive quantities, not given by product of local and quasi-local charges, can in principle exist. These terms, included in the extra contribution $\mathcal{R}$ in (6), would give an extra contribution to the full Onsager coefficients $\mathfrak{L}_{k k}$.

The second lower bound on the spin/charge diffusion constant in the zero magnetization sector/half-filling has been proposed in 33 and further studied in 25, 45]. It corresponds to the curvature of the Drude weight with respect to the magnetization $\left\langle S^{z}\right\rangle$ filling $\nu(T, h)=$ $4 T\left\langle S^{z}\right\rangle$ as $\mathfrak{L}_{s, s} \geq \partial_{\nu}^{2} D(h) / v_{\mathrm{LR}}$. As a consequence of the spin flip symmetry in the considered examples, one of the normal modes in our expression for the diffusion constant (13) should be magnetization $N_{i}=S^{z}$. Since the velocity of magnetization normal mode vanishes, the lower bound can be reproduced by replacing the velocity in our expression (13) with their upper bound $v_{\text {LR }}$.

\section{Diffusion in integrable systems}

Following recent developments in the hydrodynamic description of integrable systems 23 , 31, 46, 46 51], the transformation $R$ as well as other transport matrices such as $A, B$, and $G$ can be computed exactly. In integrable systems normal modes are stable quasi-particle excitations that fully describe the thermodynamics and hydrodynamics of the system 52,53 . An expression for the $\mathfrak{L}_{i j}$ matrix has been found in [31] by exploiting integrability techniques. It is not hard to see that our result reproduces this expression exactly $\mathfrak{L}_{i j}^{c} \equiv \mathfrak{L}_{i j} \mathrm{~S} 2.3$. Notice that the same applies for the diffusion matrices of hard rod gases [19]. We can therefore conclude that diffusive transport in integrable models and hard rods gases is given purely by the dispersion of their ballistic modes.

Our result also gives a simple explanation of the curious "magic formula", which was established rigorously at infinite temperatures [54], and numerically at finite temperatures. The "magic formula" relates the curvature of self-Drude weight $D_{s, s}^{\text {self }}=\int \mathrm{d} t\left\langle j_{s}(0, t) j_{s}(0,0)\right\rangle^{c}$ where $j_{s}$ is the spin (or charge) current, with the spin diffusion constant $\mathfrak{D}_{s, s}=\mathfrak{L}_{s, s} / C_{s, s}=$ $\partial_{\nu}^{2} D_{s, s}^{\text {self }}(\nu)$. This is now readily understood by noticing that the curvature of the self-Drude 
weight is equivalent to equation (11) with $\mathfrak{q}_{j}=S^{z}$ and $j_{k}=j_{s}$, and that in integrable models $\mathfrak{L}_{k l}^{c} \equiv \mathfrak{L}_{k l}$.

\section{NLFHD and super-diffusion:}

NLFHD is based on expanding the expectation values of the extensive currents in terms of the local conservation laws up to the second-order and adding phenomenological diffusion constants and white noise terms related by the fluctuation-dissipation relation $28,29,34,35$. Since the phenomenological diffusion constant and white noise affects diffusive processes, NLFHD has no predictive power on diffusive scales. However, in the presence of a single conserved charge one obtains a noisy Burgers' equation, provided that the current has an overlap with the square of the charge. Burgers' equation is known to belong to the KPZ universality class with dynamical exponent $z=\frac{3}{2}, 36,55$. . This implies that diffusion constant diverges $\mathfrak{L}=\infty$. Similar behavior can be identified in the presence of multiple modes, and detailed analysis reveals a plethora of new super-diffusive universality classes arising in the presence of appropriate multi-mode couplings [35]. This behavior can be understood within our framework, since the self-coupling term corresponds to the presence of non-zero matrix elements with degenerate velocities $(12)$, and results in the divergence of the convective Onsager coefficients (11). More specifically, the diffusion constant of a mode diverges whenever the mode coupling matrix $G_{i}^{j k}$ has non-zero elements for at least two modes with degenerate velocities. Such situations were typically exempted from past NLFHD applications, where the non-degeneracy of velocities was usually assumed in order to justify the mode decoupling assumption. Such situations are, however, expected to be important in integrable chains, where super-diffusion can also be observed for the spin or charge degrees of freedom close to half-filling $[56[59]$.

\section{Conclusion}

We have introduced an operatorial expansion of the currents in a many-body system in terms of hydrodynamical densities of conservation laws in generic stationary states. We have shown that the second-order terms of this expansion give rise to a finite contribution to the Onsager matrix and therefore to the diffusion constants of the system. Our framework unifies previous results on diffusive transport in one dimension and shows that in integrable systems convective contributions saturate the exact diffusion constants, demonstrating that other mechanisms are absent. Nevertheless, convective contributions account only for a part of the full diffusion constant in classical probabilistic dynamical systems [21, 30], and are completely absent in the spin chain with strong dephasing [60]. These partial results call for clarification of which contributions are non-vanishing in certain systems and whether generic Hamiltonian systems support non-trivial quadratic charges that are not included in the convective part of diffusion.

For the case of integrable models we provided another non-trivial verification of the expression for the diffusion constants and proved the so-called magic formula. Moreover, we have shown how the diffusion constant of a certain charge can diverge whenever there is degeneracy of the velocity of the modes. While this mechanism is valid for generic systems, it might prove to be useful to explain the emerging KPZ super-diffusive dynamics of spin and 
charge in quantum and classical isotropic chains [54,61,62]. In these models the spin/charge mode at half-filling has zero velocity and it constitutes an accumulation point for all other modes with finite velocities [63] that approach zero as $v_{i} \equiv v_{\theta, a} \sim a^{-1}$ 45, 61] for any $\theta$. An interesting direction for future research is to develop these ideas on a more rigorous footing.

While we here explored the effects of convective modes on the Onsager matrix, they can also contribute on the level of higher-order cumulants of the currents. Our construction indicates that the contributions are hierarchically ordered with respect to the order of the cumulant, analogously to the BBGKY hierarchy in the Boltzmann equation [64, 65]. Finally, our expansion can also be applied in higher dimensional systems, where normal diffusion is typically found. It would be interesting to compare our convective contribution with the known results [66].

Acknowledgement: We thank B. Doyon for numerous fruitful exchanges, and would like to refer to his complementary publication [40]. We would also like to thank D. Bernard, B. Doyon, E. Ilievski for useful comments on the manuscript. J.D.N. is supported by Research Foundation Flanders (FWO). TY acknowledges the support by Takenaka Scholarship Foundation, and hospitality at Tokyo Institute of Technology.

\section{S1 Extra details on derivation of hydrodynamics from the lat- tice}

\section{S1.1 Correlations on super-lattice}

In order to identify the effects of multi-point correlation functions on transport coefficients we consider an infinitely large spin chain, which we divide into the parts of equal length $\Delta x=\ell$, i.e. the hydrodynamical cells. We will denote the macroscopic coordinate which determines the position on this supper-lattice by $\chi, \chi=x \times \ell$.

First of all, we will show that multi-point correlation functions of hydrodynamic density of the local operator and conservation laws scale linearly with the size of the hydrodynamic cell, if all of the operators are located within the same hydrodynamic cell, and are at most constant otherwise. This result is necessary to establish that the contributions from the beyond nearest neighboring cells, and higher orders in the expansion cannot contribute to the Onsager matrix.

The first part of the result follows directly from the clustering property of GGE's and the fact that the cells that are not nearest neighbors get separated with increasing $\ell$. The second part of the result, follows from simply noticing that the higher point connected correlators can be interpreted as a derivative of expectation values, which scales linearly with the size of the cell.

The hydrodynamical density $\mathfrak{o}(\chi)$ is given by

$$
\mathfrak{o}(\chi)=\sum_{i=\chi-\ell / 2+1}^{\chi+\ell / 2} o(i),
$$


in terms of the quasilocal operator

$$
o(i)=\sum_{k=0}^{\infty} o_{[i, i+k]} .
$$

Quasilocality means that the spectral norm is upper bounded by

$$
\left\|o_{[i, i+k]}\right\|<\alpha_{1} \times \exp \left(-\eta_{1} k\right)
$$

for some $\alpha, \zeta>0$, and the $C^{*}$ norm $\|\bullet\|$. The operators $o_{[i, i+k]}$, are supported on the sublattice $[i, i+k]$, and act as an identity operator everywhere else.

The multi-point connected correlation function is defined as

$$
\left\langle\mathfrak{o}(\chi) \mathfrak{q}^{1}\left(\chi_{1}\right) \cdots \mathfrak{q}^{N}\left(\chi_{N}\right)\right\rangle^{c} \equiv \frac{\partial^{N}\langle\mathfrak{o}(\chi)\rangle}{\partial \beta^{1}\left(\chi_{1}\right) \cdots \partial \beta^{N}\left(\chi_{N}\right)},
$$

with the expectation value $\langle\bullet\rangle=\frac{\operatorname{tr}(\bullet \rho(\underline{\beta}(\chi)))}{\operatorname{tr}(\rho(\underline{\beta}(\chi)))}$ in a generalized, locally thermalized state $\rho(\underline{\beta}(\chi))=\exp \left(\beta^{i}(\chi) \mathfrak{q}_{i}(\chi)\right)$. Assuming that all of conserved densities commute up to the boundary terms, the two point connected correlation function $\langle\mathfrak{a}, \mathfrak{q}\rangle=\langle\mathfrak{a} \mathfrak{q}\rangle^{c}$. We are considering a set of states $\rho$ which satisfy exponential clustering

$$
\left\langle a_{[i, j]} b_{[k, l]}\right\rangle^{c}<\alpha_{2} \exp \left(-\eta_{2}|j-k|\right)\left\|a_{[i, j]}\right\| \times\left\|b_{[k, l]}\right\|,
$$

where we assumed the ordering $i \leq j \leq k \leq l$. It is not hard to see that the pairs of hydrodynamical operators decay exponentially with the distance

$$
\left|\left\langle\mathfrak{o}_{1}(\chi), \mathfrak{q}_{2}\left(\chi^{\prime}\right)\right\rangle\right| \leq \alpha_{3} \exp \left(-\eta_{3} \ell\right)
$$

if $\left|\chi-\chi^{\prime}\right|>2$, i.e. the operators are not located in the same, or the neighboring cells. In order to demonstrate the property $(\mathrm{S} 6)$ we can divide the connected correlator into two contributions

$$
\begin{gathered}
\left|\left\langle\mathfrak{o}_{1}(\chi), \mathfrak{q}_{2}\left(\chi^{\prime}\right)\right\rangle\right| \leq \sum_{i=x-\ell / 2+1}^{x+\ell / 2} \sum_{k=0}^{\ell / 2}\left|\left\langle o_{1[i, i+k]}, \mathfrak{q}_{2}\left(\chi^{\prime}\right)\right\rangle\right|+ \\
\quad+\sum_{i=x-\ell / 2+1}^{x+\ell / 2} \sum_{k=\ell / 2+1}^{\infty}\left|\left\langle o_{1[i, i+k]}, \mathfrak{q}_{2}\left(\chi^{\prime}\right)\right\rangle\right|=s_{1}+s_{2}
\end{gathered}
$$

In order to upper bound the second term $s_{2}$ we can use the trivial bound

$$
\langle a, b\rangle \leq\|a\| \times\|b\|
$$

and quasilocality $(\mathrm{S} 3)$

$$
s_{2} \leq \text { Const. } \times \ell^{2} \exp \left(-\eta_{1} \ell / 2\right) .
$$

In order to evaluate the first term, we take into account that $\left|\chi-\chi^{\prime}\right|>2$, implying that the minimal distance between the operators in $\mathfrak{o}_{1}$ and densities $\mathfrak{q}_{2}$ is $\frac{\ell}{2}$. This enables us to show that

$$
s_{1} \leq \text { Const. } \times \ell^{3} \exp \left(-\eta_{2} \ell / 2\right) .
$$

Now we proceed to show that the contribution from the neighboring cell is sub-polynomial in the size of hydrodynamic cell $\ell$

$$
\left|\left\langle\mathfrak{o}_{1}(\chi), \mathfrak{q}_{2}(\chi+1)\right\rangle\right| \leq \text { Const. } \times \ell^{\kappa}
$$


for arbitrary $\kappa$. We divide the sum into five parts

$$
\begin{gathered}
\left|\left\langle\mathfrak{o}_{1}(\chi), \mathfrak{q}_{2}(\chi+1)\right\rangle\right| \leq \\
\leq \sum_{i=x-\ell / 2+1}^{x+\ell / 2-\ell^{\kappa_{1}}}\left(\sum_{k=0}^{\ell^{\kappa_{2}}}\left|\left\langle o_{1[i, i+k]}, \mathfrak{q}_{2}(\chi+1)\right\rangle\right|+\sum_{k=\ell^{\kappa_{2}}}^{\infty}\left|\left\langle o_{1[i, i+k]}, \mathfrak{q}_{2}(\chi+1)\right\rangle\right|\right)+ \\
+\sum_{i=x+\ell / 2-\ell^{\kappa_{1}+1}}^{x+\ell / 2} \sum_{k=0}^{\ell^{\kappa_{2}}}\left(\sum_{j=x+\ell / 2+1}^{x+\ell / 2+\ell^{\kappa_{1}}}\left|\left\langle o_{1[i, i+k]}, q_{2, j}\right\rangle\right|+\sum_{j=x+\ell / 2+\ell^{\kappa_{1}}}^{x+3 \ell / 2}\left|\left\langle o_{1[i, i+k]}, q_{2, j}\right\rangle\right|\right)+ \\
+\sum_{i=x+\ell / 2-\ell^{\kappa_{1}+1}}^{x+\ell / 2} \sum_{k=\ell^{\kappa_{2}}}^{\infty}\left|\left\langle o_{1[i, i+k]}, \mathfrak{q}_{2}(\chi+1)\right\rangle\right|=s_{1}+s_{2}+s_{3}+s_{4}+s_{5},
\end{gathered}
$$

with $0<\kappa_{2}<\kappa_{1}$. Similarly as before we can lower bound the second sum by using a trivial lower bound (S7) and quasilocality

$$
s_{2}, s_{5} \leq \text { Const. } \times \ell^{2} \exp \left(-\eta_{1} \ell^{\kappa_{2}}\right) .
$$

Using similar arguments as before, we get

$$
s_{1}, s_{4} \leq \text { Const. } \times \ell^{\kappa_{2}+2} \exp \left(-\eta_{2}\left(\ell^{\kappa_{1}}-\ell^{\kappa_{2}}\right)\right) .
$$

Using a trivial bound (S7), we can upper bound $s_{3}$ by

$$
s_{3} \leq \text { Const. } \times \ell^{\kappa_{2}+2 \kappa_{1}} .
$$

Since $\kappa=2 \kappa_{1}+\kappa_{2}$ can be arbitrarily small, we arrive at the result (S10).

We are now in the position to prove extensivity and orthogonality of arbitrary multipoint connected correlation function on the super-lattice

$$
\left\langle\mathfrak{o}_{1}\left(\chi_{1}\right) \mathfrak{q}_{2}\left(\chi_{2}\right) \cdots \mathfrak{q}_{N}\left(\chi_{N}\right)\right\rangle^{c}=\ell\left(\left\langle\mathfrak{o}_{1}\left(\chi_{1}\right) \cdots \mathfrak{q}_{N}\left(\chi_{1}\right)\right\rangle_{n}^{c} \delta \chi_{1} \chi_{2} \delta_{\chi_{2} \chi_{3}} \cdots \delta_{\chi_{N-1} \chi_{N}}+\mathcal{O}\left(\ell^{-1}\right)\right)
$$

The $N$-point connected correlation function is at most extensive, since the expectation value $\langle\mathfrak{o}\rangle$ is proportional to the volume of the cell $\propto \ell$. And the $N$-point correlation function corresponds to the $N-1$ point derivative of the expectation value. In the absence of phase transitions divergences are absent, implying extensivity of $N$-point correlation function.

If the distance between at least two operators in the connected correlation function is $\left|\chi-\chi^{\prime}\right|>2$, the correlation function vanishes exponentially in the hydrodynamical limit $\ell \rightarrow \infty$. Let's assume that $\mathfrak{o}_{i}(\chi)$ and $\mathfrak{q}_{j}(\chi)$ do not occupy the same or the neighboring cells. Using (S6) we have that

$$
\left|\left\langle\mathfrak{o}_{i}(\chi), \mathfrak{q}_{j}\left(\chi^{\prime}\right)\right\rangle\right| \leq \exp \left(-\eta_{2}\left(\beta_{1}(\chi), \ldots, \beta_{N}\left(\chi_{N}\right)\right) \ell\right),
$$

and taking the derivatives produces at most polynomial factor $\ell^{N-2}$.

A technical result that we will need in next section is a hydrodynamical decomposition of the two point connected correlation function of squares of hydrodynamical densities into the products of two point functions of local hydrodynamical densities

$$
\left\langle\delta \mathfrak{q}_{1} \delta \mathfrak{q}_{2}, \delta \mathfrak{q}_{3} \delta \mathfrak{q}_{4}\right\rangle=\left\langle\delta \mathfrak{q}_{1}, \delta \mathfrak{q}_{3}\right\rangle\left\langle\delta \mathfrak{q}_{2}, \delta \mathfrak{q}_{4}\right\rangle+\left\langle\delta \mathfrak{q}_{1}, \delta \mathfrak{q}_{4}\right\rangle\left\langle\delta \mathfrak{q}_{2}, \delta \mathfrak{q}_{3}\right\rangle+\mathcal{O}(\ell)
$$

which we are going to prove only at infinite temperature but holds for any clustering density matrix $\rho$. The result can be inferred by explicitly decomposing the four point function into the sum of local terms

$$
\left.\left\langle\delta q_{1} \delta q_{2} \delta q_{3} \delta q_{4}\right)\right\rangle_{\infty}=\sum_{\alpha_{1}, \alpha_{2}, \alpha_{3}, \alpha_{4}} \sum_{r_{1}, r_{2}, r_{3}, r_{4}} \frac{\operatorname{tr}\left(\delta q_{1}^{\left[\alpha_{1}, \alpha_{1}+r_{1}\right]} \delta q_{2}^{\left[\alpha_{2}, \alpha_{2}+r_{2}\right]} \delta q_{3}^{\left[\alpha_{3}, \alpha_{3}+r_{3}\right]} \delta q_{4}^{\left[\alpha_{4}, \alpha_{4}+r_{4}\right]}\right)}{\operatorname{tr}(\mathbb{1})}
$$


In order to evaluate the above sum we divide it into two parts. The first part corresponds to the case in which at least three of the densities overlap, and thus form a connected cluster. Such term will yield a finite contribution, only if all four densities form a connected cluster. If the largest support of the density in the above sum is $r_{\beta}=\max \left(r_{1}, r_{2}, r_{3}, r_{4}\right)$, then the absolute value of the sum over $\alpha_{k}, k \neq \beta$ of such terms can be upper bounded by

$$
\begin{gathered}
\sum_{k \neq \beta} \sum_{\alpha_{k}}\left|\frac{\operatorname{tr}\left(\delta q_{1}^{\left[\alpha_{1}, \alpha_{1}+r_{1}\right]} \delta q_{2}^{\left[\alpha_{2}, \alpha_{2}+r_{2}\right]} \delta q_{3}^{\left[\alpha_{3}, \alpha_{3}+r_{3}\right]} \delta q_{4}^{\left[\alpha_{4}, \alpha_{4}+r_{4}\right]}\right)}{\operatorname{tr}(\mathbb{1})}\right| \leq \\
\leq r_{\beta}^{3} \exp \left(-\eta\left(r_{1}+r_{2}+r_{3}+r_{4}\right)\right), \quad \eta>0 .
\end{gathered}
$$

Furthermore we can upper bound $r_{\beta}<\left(r_{1}+r_{2}+r_{3}+r_{4}\right)$, implying that the summing over $r_{1}, r_{2}, r_{3}, r_{4}$ yields a finite contribution. The only summation that remains is the one over $\beta$. This produces a factor which is proportional to the cell size $\ell$.

In order to consider remaining contributions, we have to take into account the cases, where none of the operators overlap at any point, and the case where exactly two operators overlap. In the first case the contribution automatically vanishes due to the tracelesness of $\delta \mathfrak{q}$. In order to compactly represent the second contribution we perform the expansion of the product of the two point correlation function

$$
\begin{gathered}
\left\langle\delta \mathfrak{q}_{1}, \delta \mathfrak{q}_{3}\right\rangle\left\langle\delta \mathfrak{q}_{2}, \delta \mathfrak{q}_{4}\right\rangle= \\
=\sum_{\alpha_{1}, \alpha_{2}, \alpha_{3}, \alpha_{4}} \sum_{r_{1}, r_{2}, r_{3}, r_{4}} \frac{\operatorname{tr}\left(\delta q_{1}^{\left[\alpha_{1}, \alpha_{1}+r_{1}\right]} \delta q_{2}^{\left[\alpha_{2}, \alpha_{2}+r_{2}\right]}\right) \operatorname{tr}\left(\delta q_{3}^{\left[\alpha_{3}, \alpha_{3}+r_{3}\right]} \delta q_{4}^{\left[\alpha_{4}, \alpha_{4}+r_{4}\right]}\right)}{\operatorname{tr}(\mathbb{1})^{2}} .
\end{gathered}
$$

Using equivalent arguments as above, we can show that the terms which overlap scale linearly with the system size $\ell$.

This immediately implies that the trace of the product of four extensive operators can be represented as the sum of two point functions up to corrections of the order $\mathcal{O}(\ell)$, since the contributions from the operators corresponding to the overlapping of the support of two pairs of operators in the four point function produces exactly the contributions of the form (S18) up to the overlaping terms. This results in the decomposition (S16). Note that this expression is divided by $\ell^{2}$, and disregarded terms result in the $\frac{1}{\ell}$ correction to the diffusion constant.

\section{S1.2 Consistency condition}

While in the main text a physical argument for the form of expansion was given, the coefficients can be obtained from the consistency condition.

The starting point is the local expansion in which we assume that the higher order terms in expansion

$$
\delta \mathfrak{o}=c^{i} \delta \mathfrak{q}_{i}+\frac{c^{i j}}{\ell} \delta\left(\mathfrak{q}_{i} \mathfrak{q}_{j}\right)+\frac{c^{i j k}}{\ell^{2}} \delta\left(\mathfrak{q}_{i} \mathfrak{q}_{j} \mathfrak{q}_{k}\right)+\ldots
$$

take the form that reproduces the connected correlation function, i.e. $\left\langle\mathfrak{o}, \delta\left(\mathfrak{q}_{i_{1}} \cdots \mathfrak{q}_{i_{k}}\right)\right\rangle \equiv$ $\left\langle\mathfrak{o}_{1} \mathfrak{q}_{i_{1}} \cdots \mathfrak{q}_{i_{k}}\right\rangle^{c} \leq$ Const. $\times \ell$. In order for the expansion (S19) to be consistent, we have to require that the expansion $\delta \mathfrak{o}_{k}(\chi)=\left(c_{k}^{i} \delta \mathfrak{q}_{i}(\chi)+\frac{c_{k}^{i j}}{\ell} \delta \mathfrak{q}_{i}(\chi) \delta \mathfrak{q}_{j}(\chi)+\ldots\right)$ is consistent on the level of two point functions. In particular the leading order of the two point correlation function scales linearly with $\ell$ and reads

$$
c_{1}^{i}\left\langle\mathfrak{o}_{2}, \delta \mathfrak{q}_{j_{2}}\right\rangle=c_{1}^{i} c_{2}^{j}\left\langle\delta \mathfrak{q}_{i}, \delta \mathfrak{q}_{j}\right\rangle
$$


Note also that $\delta\left(\mathfrak{q}_{i} \mathfrak{q}_{j}\right)=\delta \mathfrak{q}_{i} \delta \mathfrak{q}_{j}$. Demanding that the above equality is satisfied for all operators $\mathfrak{o}_{1}$ (setting $c^{i_{1}^{\prime}}=\delta_{i_{1}, k}$ in particular) the coefficients can be obtained easily by inverting the relation $\mathrm{S} 20$

$$
c_{1}^{i}=\lim _{\ell \rightarrow \infty} \frac{1}{\ell} C^{i j}\left\langle\mathfrak{o}, \delta \mathfrak{q}_{j}\right\rangle
$$

After solving the leading order equations, this contribution should be subtracted in expansion (S19) in order to eliminate the terms corresponding to the first order. The equations for the second order can then be obtained by taking the limit $\ell \rightarrow \infty$, which removes the higher order terms

$$
\frac{c_{1}^{i j}}{\ell}\left\langle\mathfrak{o}^{-}, \delta \mathfrak{q}_{i} \delta \mathfrak{q}_{j}\right\rangle=\frac{c_{1}^{i j} c_{2}^{k l}}{\ell^{2}}\left\langle\delta \mathfrak{q}_{i} \delta \mathfrak{q}_{j}, \delta \mathfrak{q}_{k} \delta \mathfrak{q}_{l}\right\rangle
$$

Taking into account the decomposition of the four point function $\left\langle\delta \mathfrak{q}_{i} \delta \mathfrak{q}_{j}, \delta \mathfrak{q}_{k} \delta \mathfrak{q}_{l}\right\rangle$ into two point functions (S16) and symmetry property of coefficients $c_{r}^{i j}=c_{r}^{j i}$ for $r \in\{1,2\}$, one readily recovers the result

$$
c_{2}^{i j}=\frac{1}{2} \lim _{\ell \rightarrow \infty} \frac{1}{\ell} C^{i k} C^{j l}\left\langle\mathfrak{o}^{-}, \delta \mathfrak{q}_{k} \delta \mathfrak{q}_{l}\right\rangle
$$

by following the first order prescription.

\section{S2 Extra Derivations of equations}

\section{S2.1 Derivation of Drude weights}

As noted in the main text in order to derive the Drude weight and diffusion constant, we will use the dynamics of normal modes on Euler scale

$$
\mathfrak{n}_{i}(\chi, \tau)=\sum_{\chi^{\prime}} \frac{1}{2 \pi} \int_{-\pi}^{\pi} \mathrm{d} k e^{i k\left(\chi-\chi^{\prime}\right)-i \omega_{i}(k) \tau} \mathfrak{n}_{i}\left(\chi^{\prime}\right)+\ldots,
$$

up to $\frac{1}{\ell}$ corrections, and the hydrodynamic expansion

$$
\begin{aligned}
& \delta \mathfrak{o}(\chi)=\left(\partial_{\mathrm{q}_{i}(\chi)}\langle\delta \mathfrak{o}(\chi)\rangle\right) \delta \mathfrak{q}_{i}(\chi)+ \\
& +\frac{1}{2}\left(\partial_{\mathbf{q}_{j}(\chi)} \partial_{\mathrm{q}_{i}(\chi)}\langle\delta \mathfrak{o}(\chi)\rangle\right) \delta \mathfrak{q}_{i}(\chi) \delta \mathfrak{q}_{j}(\chi)+\mathcal{R},
\end{aligned}
$$

for the current.

In order to establish how the current expansion works in actual computations, we will apply it to the computation of Drude weight. The Drude weights $D_{i, j}$ are defined by

$$
D_{i, j}=\lim _{\tau \rightarrow \infty} \frac{1}{2 \tau \ell} \sum_{\chi} \int_{-\tau}^{\tau} \mathrm{d} \tau^{\prime}\left\langle\mathfrak{j}_{i}\left(0, \tau^{\prime}\right), \mathfrak{j}_{j}(\chi, 0)\right\rangle .
$$

For computing this object, we need the first order in the hydrodynamic expansion (S25) only. 
Inserting this term into $(\mathrm{S} 26)$ gives

$$
\begin{aligned}
D_{i, j} & =\lim _{\tau \rightarrow \infty} \frac{1}{2 \tau \ell} \sum_{\chi} \int_{-\tau}^{\tau} \mathrm{d} \tau^{\prime} \frac{\partial\left\langle\mathfrak{j}_{i}(0,0)\right\rangle}{\partial \mathfrak{q}_{k}(0,0)}\left\langle\mathfrak{q}_{k}\left(0, \tau^{\prime}\right), \mathfrak{j}_{j}(\chi, 0)\right\rangle= \\
& =\lim _{\tau \rightarrow \infty} \frac{1}{2 \tau \ell} \sum_{\chi} \int_{-\tau}^{\tau} \mathrm{d} \tau^{\prime} \frac{1}{\ell} C^{k l}\left\langle\mathfrak{j}_{i}(0,0), \mathfrak{q}_{k}(0,0)\right\rangle\left\langle\mathfrak{q}_{l}\left(0, \tau^{\prime}\right), \mathfrak{j}_{j}(\chi, 0)\right\rangle= \\
& =\left(B C^{-1} B\right)_{i, j},
\end{aligned}
$$

where we used the relation $\partial_{\beta^{i}(x)}=\ell C_{i j} \partial_{\mathrm{q}_{j}(x)}$, which follows from the clustering property (S14). To go from the second to the third line, one should notice that due to the homogeneity of the stationary state, the space dependence of the current $\chi$ can be moved to the charge $\mathfrak{q}_{l}\left(0, \tau^{\prime}\right)$. Summing over the spatial coordinate $\chi$ results in a conserved quantity, allowing us to drop the time dependence $\tau^{\prime}$.

Alternatively, changing to the normal mode basis using the convention $R C R^{\mathrm{T}}=1$, one can write the Drude weight as

$$
D_{i, j}=\left\langle j_{i}, N^{k}\right\rangle\left\langle N_{k}, j_{j}\right\rangle
$$

where $N_{i}=R_{i}^{j} Q_{j}$ is the total charge in the normal mode basis. Note that in the computation involving the current expansion (6), we always take the homogeneous limit of the averages only at the end of computations.

\section{S2.2 Derivation of the Onsager matrix}

In this section, we present a derivation of the convective contribution to the Onsager matrix $\mathfrak{L}_{u, v}^{c}$, and hence the diffusion constant. Recall that the Onsager matrix is given by the following expression

$$
\mathfrak{L}_{u, v}=\int \mathrm{d} t\left(\sum_{x}\left\langle j_{u}(x, t) j_{v}(0,0)\right\rangle^{c}-D_{u, v}\right),
$$

where $D_{u, v}$ corresponds to the Drude weight. In order to better understand how each term scale with $\ell$, let us rewrite it in terms of the hydrodynamic current $j$

$$
\mathfrak{L}_{u, v}=\int \mathrm{d} \tau\left(\sum_{\chi}\left\langle\mathfrak{j}_{u}(\chi, \tau), \mathfrak{j}_{v}(0,0)\right\rangle-D_{u, v}\right) .
$$

We will take the hydrodynamic limit $\ell \rightarrow \infty$ only in the end. In order to study corrections to the Euler scale hydrodynamics, which corresponds to the nonzero Drude weight, and can be interpreted as a consequence of the first term in the expression for the current (S25), it proves useful to consider a part of the current that characterizes the sub-Euler contribution

$$
\mathfrak{j}_{i}^{-}(\chi, \tau)=\mathfrak{j}_{i}(\chi, \tau)-\left(\partial_{\mathbf{q}_{j}(\chi)}\left\langle\mathfrak{j}_{i}(\chi)\right\rangle\right) \mathfrak{q}_{j}(\chi, \tau) .
$$

The Onsager matrix now reads

$$
\mathfrak{L}_{u, v}=\int \mathrm{d} \tau \sum_{\chi}\left\langle\mathfrak{j}_{u}^{-}(\chi, \tau), \mathfrak{j}_{v}^{-}(0,0)\right\rangle .
$$


Inserting the expression of the current into equation (S30) we obtain

$$
\begin{aligned}
\mathfrak{L}_{u, v}= & \sum_{\chi} \frac{\ell}{2} \int \mathrm{d} \tau\left(\partial_{\mathbf{q}_{j}(0,0)} \partial_{\mathbf{q}_{i}(0,0)}\left\langle\mathfrak{j}_{v}(0,0)\right\rangle\right)\left(\left\langle\mathfrak{q}_{i}(0,0) \mathfrak{q}_{j}(0,0) \mathfrak{j}_{u}(\chi, \tau)\right\rangle_{n}^{c}-\right. \\
& \left.-A_{u}^{k}\left\langle\mathfrak{q}_{i}(0,0) \mathfrak{q}_{j}(0,0) \mathfrak{q}_{k}(x, t)\right\rangle_{n}^{c}\right)= \\
= & \frac{\ell}{2}\left(\partial_{\mathbf{q}_{j}(0,0)} \partial_{\mathbf{q}_{i}(0,0)}\left\langle\mathfrak{j}_{v}(0,0)\right\rangle\right)\left(M_{i j}^{j_{u}}-A_{u}^{k} M_{i j}^{\mathfrak{q}_{k}}\right),
\end{aligned}
$$

where we defined

$$
M_{i j}^{\mathfrak{o}}=\sum_{\chi} \int \mathrm{d} \tau\left\langle\mathfrak{q}_{i}(0, \tau) \mathfrak{q}_{j}(0, \tau) \mathfrak{o}(\chi, 0)\right\rangle_{n}^{c}
$$

for an arbitrary hydrodynamic operator $\mathfrak{o}$, and introduced a normalized connected correlation function $\left\langle\mathfrak{q}_{i}(0,0) \mathfrak{q}_{j}(0,0) \mathfrak{j}_{u}(\chi, \tau)\right\rangle_{n}^{c} \equiv \frac{1}{\ell}\left\langle\mathfrak{q}_{i}(0,0) \mathfrak{q}_{j}(0,0) \mathfrak{j}_{u}(\chi, \tau)\right\rangle^{c}$. To proceed, let us first deal with a building block $M_{i j}^{\mathrm{o}}$ and rewrite it in terms of normal modes. Using the solution of $\mathfrak{n}(\chi, \tau)(\mathrm{S} 24)$, we have

$$
\begin{aligned}
M_{i j}^{\mathfrak{o}} & =\left(R^{-1}\right)_{i}^{i^{\prime}}\left(R^{-1}\right)_{j}^{j^{\prime}} \times \\
& \times \sum_{\chi} \int \mathrm{d} \tau \int_{-\pi}^{\pi} \frac{\mathrm{d} k}{2 \pi} \frac{\mathrm{d} k^{\prime}}{2 \pi} e^{-\mathrm{i}\left(k+k^{\prime}\right) \chi} e^{\left.-\mathrm{i}\left(\omega_{i^{\prime}}(k)\right)+\omega_{j^{\prime}}\left(k^{\prime}\right)\right) \tau}\left\langle\mathfrak{n}_{i^{\prime}}(0,0) \mathfrak{n}_{j^{\prime}}(0,0) \mathfrak{o}(0,0)\right\rangle_{n}^{c}
\end{aligned}
$$

Now using that $\sum_{\chi=-\infty}^{\infty} e^{\mathrm{i} k \chi}=2 \pi \delta(k)$, we get

$$
M_{i j}^{\mathfrak{o}}=\left(R^{-1}\right)_{i}^{i^{\prime}}\left(R^{-1}\right)_{j}^{j^{\prime}} \int \mathrm{d} \tau \int_{-\pi}^{\pi} \frac{\mathrm{d} k}{2 \pi} e^{-\mathrm{i}\left(\omega_{i^{\prime}}(k)+\omega_{j^{\prime}}(-k)\right) \tau}\left\langle\mathfrak{n}_{i^{\prime}}(0,0) \mathfrak{n}_{j^{\prime}}(0,0) \mathfrak{o}(0,0)\right\rangle_{n}^{c}
$$

The integration over $\tau$ can be done as follows

$$
\int \mathrm{d} \tau e^{-\mathrm{i}\left(\omega_{i^{\prime}}(k)+\omega_{j^{\prime}}(-k)\right) \tau}=\frac{2 \pi \delta(k)}{\left|v_{i^{\prime}}-v_{j^{\prime}}\right|},
$$

which allows us to obtain a compact expression of $M_{i j}^{\mathfrak{o}}$

$$
\begin{aligned}
M_{i j}^{\mathfrak{o}} & =\left(R^{-1}\right)_{i}^{i^{\prime}}\left(R^{-1}\right)_{j}^{j^{\prime}} \frac{1}{\left|v_{i^{\prime}}-v_{j^{\prime}}\right|}\left\langle\mathfrak{n}_{i^{\prime}}(0,0) \mathfrak{n}_{j^{\prime}}(0,0) \mathfrak{o}(0,0)\right\rangle_{n}^{c}= \\
& =\left(R^{-1}\right)_{i}^{i^{\prime}}\left(R^{-1}\right)_{j}^{j^{\prime}} \frac{1}{\left|v_{i^{\prime}}-v_{j^{\prime}}\right|} R_{i^{\prime}}^{i^{\prime \prime}} R_{j^{\prime}}^{j^{\prime \prime}}\left\langle\mathfrak{q}_{i^{\prime \prime}}(0,0) \mathfrak{q}_{j^{\prime \prime}}(0,0) \mathfrak{o}(0,0)\right\rangle_{n}^{c}= \\
& =\left(R^{-1}\right)_{i}^{i^{\prime}}\left(R^{-1}\right)_{j}^{j^{\prime}} \frac{1}{\left|v_{i^{\prime}}-v_{j^{\prime}}\right|} R_{i^{\prime}}^{i^{\prime \prime}} R_{j^{\prime}}^{j^{\prime \prime}} \frac{\partial^{2}\langle o\rangle}{\partial \beta^{i^{\prime \prime}} \partial \beta^{j^{\prime \prime}}} .
\end{aligned}
$$

Notice that in the final line, the hydrodynamic observable $\mathfrak{o}(0,0)$ is replaced by the ordinary local observable $o$. We further observe that the curvature term $\frac{\partial^{2}\langle o\rangle}{\partial \beta^{i \prime} \partial \beta^{j^{\prime \prime}}}$ can be written as

$$
\frac{\partial^{2}\langle o\rangle}{\partial \beta^{i^{\prime \prime}} \partial \beta^{j^{\prime \prime}}}=\frac{\partial^{2}\langle o\rangle}{\partial \mathrm{q}_{k} \partial \mathrm{q}_{k^{\prime}}} C_{i^{\prime \prime} k} C_{j^{\prime \prime} k^{\prime}}+\frac{\partial\langle o\rangle}{\partial \mathrm{q}_{k}} \frac{\partial}{\partial \beta^{k}} C_{i^{\prime \prime} j^{\prime \prime}}
$$


according to which $\mathrm{S33}$ becomes

$$
\begin{aligned}
\mathfrak{L}_{u, v} & =\frac{\ell}{2}\left(\partial_{\mathrm{q}_{j}(0,0)} \partial_{\mathrm{q}_{i}(0,0)}\left\langle\mathbf{j}_{v}(0,0)\right\rangle\right)\left(M_{i j}^{\mathrm{j}_{u}}-A_{u}^{k} M_{i j}^{\mathfrak{q}_{k}}\right)= \\
& =\frac{\ell}{2}\left(\partial_{\mathrm{q}_{j}(0,0)} \partial_{\mathrm{q}_{i}(0,0)}\left\langle\mathbf{j}_{v}(0,0)\right\rangle\right)\left(R^{-1}\right)_{i}^{i^{\prime}}\left(R^{-1}\right)_{j}^{j^{\prime}} \frac{1}{\left|v_{i^{\prime}}-v_{j^{\prime}}\right|}\left(R^{-\mathrm{T}}\right)_{i^{\prime} k}\left(R^{-\mathrm{T}}\right)_{j^{\prime} k^{\prime}} H_{u}^{k k^{\prime}}= \\
& =\ell\left(\partial_{\mathrm{q}_{j}(0,0)} \partial_{\mathrm{q}_{i}(0,0)}\left\langle\mathbf{j}_{v}(0,0)\right\rangle\right)\left(R^{-1}\right)_{i}^{i^{\prime}}\left(R^{-1}\right)_{j}^{j^{\prime}} \frac{1}{\left|v_{i^{\prime}}-v_{j^{\prime}}\right|}\left(R^{-1}\right)_{u}^{u^{\prime}} G_{u^{\prime}}^{i^{\prime} j^{\prime}},
\end{aligned}
$$

where $H$ matrix corresponds to $H_{v}^{i j}=\ell \partial_{\mathrm{q}_{j}(0,0)} \partial_{\mathrm{q}_{i}(0,0)}\left\langle\mathbf{j}_{v}(0,0)\right\rangle$. The $G$-matrix is defined as

$$
G_{i}^{j k}=\frac{1}{2} R_{i}^{l}\left(R^{-\mathrm{T}} H_{l} R^{-1}\right)^{j k}
$$

We are finally in the position to derive the exact convective contribution to the Onsager matrix. Putting everything together, we have

$$
\mathfrak{L}_{u, v}^{c}=2\left(R^{-1} \tilde{G}^{2} R^{-\mathrm{T}}\right)_{u v},
$$

where

$$
\tilde{G}_{i j}^{2}=\frac{1}{\left|v_{i^{\prime}}-v_{j^{\prime}}\right|} G_{i i^{\prime} j^{\prime}} G_{j}^{i^{\prime} j^{\prime}}
$$

Note that the convective Onsager matrix can also be written as

$$
\mathfrak{L}_{u, v}^{c}=\left\langle j_{u}^{-} Q_{i} Q_{j}\right\rangle^{c} \mathcal{C}^{i j ; i^{\prime} j^{\prime}}\left\langle Q_{i^{\prime}} Q_{j^{\prime}} j_{v}^{-}\right\rangle^{c}=\frac{\left\langle j_{u}^{-} N_{i} N_{j}\right\rangle^{c}\left\langle N^{i} N^{j} j_{v}^{-}\right\rangle^{c}}{2\left|v_{i}-v_{j}\right|},
$$

where

$$
\mathcal{C}^{i j ; i^{\prime} j^{\prime}}=\frac{1}{2\left|v_{i^{\prime \prime}}-v_{j^{\prime \prime}}\right|} R^{i^{\prime \prime} i} R^{j^{\prime \prime} j} R_{i^{\prime \prime}}^{i^{\prime}} R_{j^{\prime \prime}}^{j^{\prime}}
$$

\section{S2.3 Derivation of the $G$-tensor and diffusion in integrable systems}

In integrable systems, quantum and classical ones, (Lieb-Liniger model, integrable spin chains, classical and quantum integrable field theories) as well as in gases of hard rods, thermodynamics can be systematically studied by thermodynamic Bethe ansatz (TBA). These models share the property that their dynamics is completely fixed by the 2-body scattering shift $T_{i j}$, which also provides the dressing for the thermodynamic functions. Dressing denotes the properties of the modes which are immersed in the background with the finite density of quasiparticles. In the thermodynamic limit any stationary state, thermal or GGE, is fixed by the occupation function $n_{i}=\left\langle\mathfrak{n}_{i}\right\rangle \sqrt{\chi_{i}} / \rho_{i}^{\text {tot }}$ where the total density of states is given in terms of the occupations via an integral equation $\rho_{i}^{\text {tot }}=p^{\prime}+T n \rho_{i}^{\text {tot }}$. Here $p_{i}^{\prime}$ is the bare momentum of each quasiparticle $i$. The susceptibility of each mode is given by $\chi_{i}=\rho_{i}^{\text {tot }} n_{i}\left(1-n_{i}\right)$. Moreover the group velocities which we denote by $v_{i}^{\text {eff }}=(\partial \varepsilon / \partial p)_{i}$ are again obtained by solving integral equation for the dressing of the energy $\varepsilon$ and momentum $p$. The label $i$ runs over the infinite number of distinct normal modes. In standard notations the index $i$ is labeled by the continuous parameter corresponding to rapidities $\theta$ and the discrete parameter labeling distinct quasiparticle types $s$. 
An expression for the matrix $\mathfrak{L}$ was only recently found in using techniques of integrability in $[22$. It takes the following form

$$
\mathfrak{L}_{k l}=\left(R^{-1} \frac{\rho^{\mathrm{tot}}}{\sqrt{\chi}} \tilde{\mathfrak{D}} \frac{\sqrt{\chi}}{\rho^{\mathrm{tot}}} R^{-\mathrm{T}}\right)_{k l},
$$

where $T_{i j}=T_{j i}$ is the scattering shift between modes, and $R_{i}^{j}=(1-n T)_{i}{ }^{j} / \sqrt{\chi_{i}} \cdot n_{i}$ and $\rho_{i}^{\text {tot }}$ are proportional to identity matrices. The diffusion kernel can be decomposed into diagonal and off-diagonal terms

$$
\tilde{\mathfrak{D}}_{k l}=\delta_{k l} \sum_{k^{\prime}} \chi_{k^{\prime}}\left(\frac{T_{k k^{\prime}}^{\mathrm{dr}}}{\rho_{k}^{\mathrm{tot}}}\right)^{2}\left|v_{k}-v_{k^{\prime}}\right|-\chi_{k} \frac{T_{k l}^{\mathrm{dr}} T_{l k}^{\mathrm{dr}}}{\rho_{k}^{\mathrm{tot}}}\left|v_{k}-v_{l}\right| .
$$

where $T_{i j}^{\mathrm{dr}}$ is the dressed scattering shift, given by the integral equation $T_{i j}^{\mathrm{dr}}-T_{i k} n_{k} T_{k j}^{\mathrm{dr}}=T_{i j}$. The dressed scattering phase shift $T^{\mathrm{dr}}=(1-T n)^{-1} T$ can be thought of as a length of the jump of the quasi-particle upon scattering with another quasi-particle, if both of them are immersed in a thermal background [23,50. This expression provides the diffusion constants of generic integrable chain, comprising systems of classical hard rods and spin chains 22, 31 .

In order to derive this result using our expression

$$
\mathfrak{L}_{k l}^{c}=2\left(R^{-1} \tilde{G}^{2} R^{-\mathrm{T}}\right)_{k l},
$$

we need to derive an explicit form of $G$-matrix in integrable systems. We will show that it reads

$$
G_{i}^{k l}=\delta_{i}^{k} \mathfrak{g}_{i}^{l}+\delta_{i}^{l} \mathfrak{g}_{i}^{k}
$$

where

$$
\mathfrak{g}_{i}^{k}=\frac{T_{i k}^{\mathrm{dr}} \sqrt{\chi_{k}}\left(v_{k}-v_{i}\right)}{2 \rho_{i}^{\mathrm{tot}}} .
$$

The convective coefficients in the normal mode basis is

$$
\tilde{G}_{i k}^{2}=\delta_{i k} \sum_{i^{\prime}} \frac{2}{\left|v_{i}-v_{i^{\prime}}\right|} \mathfrak{g}_{i}^{i^{\prime}} \mathfrak{g}_{i i^{\prime}}+\frac{2}{\left|v_{i}-v_{k}\right|} \mathfrak{g}_{i}^{k} \mathfrak{g}_{k i}
$$

To see that (S51) when plugged into $(\mathrm{S} 48)$ reproduces $(\mathrm{S} 46)$, it is enough to check

$$
\left(\frac{\rho^{\mathrm{tot}}}{\sqrt{\chi}} \tilde{\mathfrak{D}} \frac{\sqrt{\chi}}{\rho^{\mathrm{tot}}}\right)_{k l}=2 \tilde{G}_{k l}^{2}
$$

which is obviously true, since the first term (diagonal) and the second term (off-diagonal) terms in (S51) precisely coincide with those in (S47) up to the factor 2 . The factor 2 is then accounted for by the factor in front of $\tilde{G}_{k l}^{2}$ above.

Now we proceed to compute the $G$-tensor for integrable systems directly from known generalized hydrodynamics (GHD) expressions [43]. Note that the Latin indices $i=(\theta, a)$ denote the pairs of quasi-momentum $\theta$ and the particle type $a$, and the calculations boils down to simple matrix-like manipulations. To reiterate, the $G$-tensor reads

$$
G_{i}=\frac{1}{2} R_{i}^{l}\left(R^{-1}\right)^{\mathrm{T}} H_{l} R^{-1}, \quad\left\{\begin{array}{l}
R A R^{-1}=\operatorname{diag}\left(v^{\mathrm{eff}}\right) \\
H_{i}{ }^{j k}=\frac{\partial A_{i}{ }^{j}}{\partial \rho_{k}} \\
R C R^{\mathrm{T}}=1 .
\end{array}\right.
$$


We first normalize $R=\hat{\mathcal{N}}(1-n T)$ accordingly to the prescription $R C R^{\mathrm{T}}=1$, using the normalization $\hat{\mathcal{N}}$. Since $C=(1-n T)^{-1} \rho(1-n)(1-T n)^{-1}$, we see that appropriate normalization is provided by

$$
\hat{\mathcal{N}}_{i}^{j}=\frac{\delta_{i}{ }^{j}}{\sqrt{\rho_{i}\left(1-n_{i}\right)}}=\frac{\delta_{i}{ }^{j}}{\sqrt{\chi_{i}}},
$$

where $\rho_{i}=\rho_{i}^{\text {tot }} n_{i}$ and $\chi_{i}=\rho_{i}\left(1-n_{i}\right)$ is the quasi-particle susceptibility. The $G$-matrix becomes

$$
G_{i}^{j k}=\frac{1}{2} \sqrt{\frac{\chi_{j} \chi_{k}}{\chi_{i}}}(1-n T)_{i}^{i^{\prime}}\left((1-n T)^{-1}\right)_{j^{\prime}}^{j} H_{i^{\prime}}^{j^{\prime} k^{\prime}}\left((1-n T)^{-1}\right)_{k^{\prime}}^{k} .
$$

Taking into account that

$$
\frac{\partial}{\partial \rho_{j}}=\frac{\partial n_{i}}{\partial \rho_{j}} \frac{\partial}{\partial n_{i}}=\frac{n_{i}}{\rho_{i}}(1-n T)_{i}^{j} \frac{\partial}{\partial n_{i}}
$$

we have

$$
G_{i}^{j k}=\frac{1}{2} \frac{n_{j}}{\rho_{j}} \sqrt{\frac{\chi_{j} \chi_{k}}{\chi_{i}}}(1-n T)_{i}^{i^{\prime}} \frac{\partial A_{i^{\prime}}^{k^{\prime}}}{\partial n_{j}}\left((1-n T)^{-1}\right)_{k^{\prime}}^{k}
$$

It is useful to decompose $\frac{\partial}{\partial n_{j}} A_{i}^{k}$ as follows

$$
\frac{\partial}{\partial n_{j}} A_{i}^{k}=W_{i}^{j k}+Z_{i}^{j k}
$$

where

$$
\begin{aligned}
W_{i}^{j k} & =\left[\frac{\partial}{\partial n_{j}}\left((1-n T)^{-1}\right)_{i}^{i^{\prime}}\right] v_{i^{\prime}}^{\mathrm{eff}}(1-n T)_{i^{\prime}}^{k}+\left((1-n T)^{-1}\right)_{i}^{i^{\prime}} v_{i^{\prime}}^{\mathrm{eff}} \frac{\partial}{\partial n_{j}}(1-n T)_{i^{\prime}}^{k}= \\
& =\left((1-n T)^{-1}\right)_{i}^{j} T^{j j^{\prime}}\left(A_{j^{\prime}}^{k}-v_{j}^{\mathrm{eff}} \delta_{j^{\prime}}^{k}\right),
\end{aligned}
$$

and

$$
Z_{i}^{j k}=\left((1-n T)^{-1}\right)_{i}^{i^{\prime}} \frac{\partial v_{i^{\prime}}^{\mathrm{eff}}}{\partial n_{j}}(1-n T)_{i^{\prime}}^{k}
$$

We first deal with the contribution coming from $W_{i^{\prime}}^{j k^{\prime}}$. Applying $(1-n T)_{i}^{i^{\prime}}$ to it and summing over $i^{\prime}$ results in

$$
(1-n T)_{i}^{i^{\prime}} W_{i^{\prime}}^{j k^{\prime}}=T_{j}^{j^{\prime}}\left(A_{j^{\prime}}^{k}-v_{j}^{\mathrm{eff}} \delta_{j^{\prime}}^{k}\right) \delta_{i}^{j} .
$$

Noting further that $A_{k}^{l}\left((1-n T)^{-1}\right)_{l}^{j}=v_{j}^{\text {eff }}\left((1-n T)^{-1}\right)_{k}^{j}$, we get

$$
\begin{aligned}
\frac{1}{2} \frac{n_{j}}{\rho_{j}}(1-n T)_{i}^{i^{\prime}} W_{i^{\prime}}{ }^{j}{ }^{\prime}\left((1-n T)^{-1}\right)_{k^{\prime}}^{k} & =T_{i}^{i^{\prime}} \frac{n_{i}}{2 \rho_{i}}\left(v_{k}^{\mathrm{eff}}-v_{i}^{\mathrm{eff}}\right)\left((1-n T)^{-1}\right)_{i^{\prime}}{ }^{\prime} \delta_{i}^{j}= \\
& =\frac{1}{2 \rho_{i}^{\text {tot }}}\left(v_{k}^{\mathrm{eff}}-v_{i}^{\mathrm{eff}}\right)\left(T^{\mathrm{dr}}\right)_{i}{ }^{k} \delta_{i}{ }^{j} .
\end{aligned}
$$

Let us next turn to the term involving $Z_{i}^{j k}$. We first recall that

$$
\frac{\partial v_{i}^{\mathrm{eff}}}{\partial n_{j}}=\frac{1}{n_{j}} \frac{\rho_{j}}{\rho_{i}}\left(v_{j}^{\mathrm{eff}}-v_{i}^{\mathrm{eff}}\right)\left((1-n T)^{-1}\right)_{i}^{j},
$$


which leads to

$$
\begin{aligned}
\frac{1}{2} \frac{n_{j}}{\rho_{j}}(1-n T)_{i}^{i^{\prime}} Z_{i^{\prime}}{ }^{k^{\prime}}\left((1-n T)^{-1}\right)_{k^{\prime}}^{k} & =\frac{1}{2 \rho_{i}}\left(v_{j}^{\mathrm{eff}}-v_{i}^{\mathrm{eff}}\right)\left((1-n T)^{-1}\right)_{i}^{j} \delta_{i}{ }^{k}= \\
& =\frac{1}{2 \rho_{i}^{\mathrm{tot}}}\left(v_{j}^{\mathrm{eff}}-v_{i}^{\mathrm{eff}}\right)\left(T^{\mathrm{dr}}\right)_{i}{ }^{j} \delta_{i}{ }^{k} .
\end{aligned}
$$

Combining the results we finally end up with

$$
\begin{aligned}
G_{i}^{j k} & =\frac{1}{2 \rho_{i}^{\mathrm{tot}}} \sqrt{\frac{\chi_{j} \chi_{k}}{\chi_{i}}}\left[\left(v_{j}^{\mathrm{eff}}-v_{i}^{\mathrm{eff}}\right)\left(T^{\mathrm{dr}}\right)_{i}^{j} \delta_{i}^{k}+\left(v_{k}^{\mathrm{eff}}-v_{i}^{\mathrm{eff}}\right)\left(T^{\mathrm{dr}}\right)_{i}{ }^{k} \delta_{i}^{j}\right]= \\
& =\frac{1}{2 \rho_{i}^{\mathrm{tot}}}\left[\sqrt{\chi_{j}}\left(v_{j}^{\mathrm{eff}}-v_{i}^{\mathrm{eff}}\right)\left(T^{\mathrm{dr}}\right)_{i}^{j} \delta_{i}^{k}+\sqrt{\chi_{k}}\left(v_{k}^{\mathrm{eff}}-v_{i}^{\mathrm{eff}}\right)\left(T^{\mathrm{dr}}\right)_{i}{ }^{k} \delta_{i}^{j}\right]= \\
& =\mathfrak{g}_{i}^{j} \delta_{i}{ }^{k}+\mathfrak{g}_{i}{ }^{k} \delta_{i}{ }^{j},
\end{aligned}
$$

where $\mathfrak{g}_{i}^{j}$ is given by $(\mathrm{S} 50)$. Observe that $\mathrm{S65}$ ) is manifestly symmetric with respect to indices $j$ and $k$.

\section{S3 Quadratic lower bound in the normal mode basis}

Here we relate the lower bound in terms of quadratic charges derived in [32] to the convective Onsager matrix $\mathfrak{L}_{k k}^{c}$. For simplicity we restrict the discussion to the infinite temperature state, however the generalization to finite temperatures should be possible by invoking exponential clustering property.

The first step in the derivation is to generalize the lower bound to multiple charges, by considering the norm of the operator $O=\frac{1}{T} \int_{0}^{T} \mathrm{~d} t J(t)-\frac{\alpha_{i \geq j}}{L} N_{i} N_{j}$, on the finite lattice of length $L$

$$
\left\langle A, B^{\dagger}\right\rangle=\frac{\operatorname{tr}\left(A B^{\dagger}\right)}{\operatorname{tr}(\mathbb{1})}-\frac{\operatorname{tr}(A)}{\operatorname{tr}(\mathbb{1})} \frac{\operatorname{tr}\left(B^{\dagger}\right)}{\operatorname{tr}(\mathbb{1})},
$$

where we take the normal modes $N_{i}$ and the extensive current operator $J=\sum_{x} j_{x}$ to be traceless and Hermitian. The fact that $\langle O, O\rangle \geq 0$ gives us the lower bound

$$
\frac{1}{T^{2}} \int_{0}^{T} \mathrm{~d} t \mathrm{~d} t^{\prime}\left\langle J(t), J\left(t^{\prime}\right)\right\rangle \geq \frac{\alpha^{i \geq j}}{L}\left\langle J, N_{i} N_{j}\right\rangle+\frac{\alpha^{i \geq j *}}{L}\left\langle J, N_{i} N_{j}\right\rangle-\frac{\alpha^{i \geq j} \alpha^{k \geq l *}}{L^{2}}\left\langle N_{i} N_{j}, N_{l} N_{k}\right\rangle
$$

Note that the three point function in the above expression is indeed equivalent to the three point connected correlation function due to the tracelesness of the operators, however the connected correlation involving four copies of normal modes corresponds to the two point connected correlation function of the terms in the brackets. In order to maximize the contribution on the right hand side we take a derivative with respect to $\alpha_{i j}^{*}$, which produces the set of equations

$$
\frac{1}{L}\left\langle J, N_{i} N_{j}\right\rangle=\frac{1}{L^{2}} \alpha^{k l}\left\langle N_{i} N_{j}, N_{l} N_{k}\right\rangle
$$

Taking into account the normal modes property

$$
\frac{\operatorname{tr}\left(N_{i} N_{j}\right)}{\operatorname{tr} \mathbb{1}}=L \times \delta_{i j},
$$


and the reduction $(\mathrm{S} 16)$, we obtain the relation

$$
\left\langle N_{i} N_{j}, N_{l} N_{k}\right\rangle=L^{2}\left(\delta_{i k} \delta_{j l}+\delta_{i j} \delta_{k l}\right)+\mathcal{O}(L),
$$

Inserting the property $(\mathrm{S} 70)$ into the set of optimization conditions $(\mathrm{S68})$ we obtain the set of coefficients

$$
\alpha_{i, j}=\left\langle J, N_{i} N_{j}\right\rangle_{n}\left(1-\frac{1}{2} \delta_{i j}\right) .
$$

This yields a lower bound

$$
\frac{1}{T^{2}} \int_{0}^{T} \mathrm{~d} t \mathrm{~d} t^{\prime}\left\langle J(t), J\left(t^{\prime}\right)\right\rangle \geq \sum_{i \geq j}\left\langle J, N_{i} N_{j}\right\rangle^{2}\left(1-\frac{1}{2} \delta_{i j}\right) .
$$

Finally the l.h.s of the above expression can be identified with the diffusion constant corresponding to the current $J 32$

$$
\frac{1}{T^{2}} \int_{0}^{T} \mathrm{~d} t \mathrm{~d} t^{\prime}\left\langle J(t), J\left(t^{\prime}\right)\right\rangle \propto D
$$

Following [32], the lower bound reads

$$
D \geq \frac{1}{8 v_{L R}}\left(\sum_{i \geq j}\left\langle J, N_{i} N_{j}\right\rangle^{2}\left(1-\frac{1}{2} \delta_{i j}\right)\right) .
$$

Note that if we take into account the upper bound on the difference of two velocities $\left|v_{i}-v_{j}\right| \leq$ $2 v_{L R}$ our prediction $\mathrm{S} 44$ overshoots this lower bound by the factor of 4 .

\section{S4 Remaining terms in operator expansion}

There are two contributions in the operatorial expansion which scale as $\frac{1}{\ell}$ that we did not account for. The first one corresponds to conserved quantities $\delta \mathfrak{q}^{(2)}$ that scale as $\ell^{2}$, and which are not simply a product of local densities or a linear combination of the products, which by assumption means that $\left\langle\delta \mathfrak{q}^{(2)}, \delta \mathfrak{q}_{i} \delta \mathfrak{q}_{j}\right\rangle=0$, implying that this contribution can be treated independently. Similarly, we can get $\frac{1}{\ell}$ scaling by choosing the first order in expansion, where one of the conserved charge densities lies in the neighboring cell of the operator which we are expanding. Once again such a contribution does not couple to the squares of local conserved densities, and can be treated on the separate footing.

Finally we should discuss higher order contributions from convective modes. First of all we conjecture that the correct expansion of local observables in terms of convective modes takes the following form

$$
\begin{aligned}
\delta \mathfrak{j}_{k}(\chi, \tau) & =\left(\partial_{\mathbf{q}_{i}(\chi)}\left\langle\delta \mathfrak{j}_{k}(\chi, \tau)\right\rangle\right) \delta \mathfrak{q}^{i}(\chi)+\frac{1}{2}\left(\partial_{\mathbf{q}_{j}(\chi)} \partial_{\mathbf{q}_{i}(\chi)}\left\langle\delta \mathfrak{j}_{k}(\chi, \tau)\right\rangle\right) \delta\left(\mathfrak{q}^{i}(\chi) \mathfrak{q}^{j}(\chi)\right)+ \\
& +\frac{1}{6}\left(\partial_{\mathbf{q}_{k}(\chi)} \partial_{\mathbf{q}_{j}(\chi)} \partial_{\mathbf{q}_{i}(\chi)}\left\langle\delta \mathbf{j}_{k}(\chi, \tau)\right\rangle\right) \delta\left(\mathfrak{q}^{i}(\chi) \mathfrak{q}^{j}(\chi) \mathfrak{q}^{k}(\chi)\right)+\ldots
\end{aligned}
$$

where the expansion satisfies the natural property

$$
\left\langle\mathfrak{o}, \delta\left(\mathfrak{q}^{i}(\chi) \mathfrak{q}^{j}(\chi) \mathfrak{q}^{k}(\chi)\right)\right\rangle=\left\langle\mathfrak{o} \mathfrak{q}^{i}(\chi) \mathfrak{q}^{j}(\chi) \mathfrak{q}^{k}(\chi)\right\rangle^{c} .
$$


This form produces a correct scaling of any multipoint connected correlation function.

Following our conjecture we now elaborate on possible higher order contributions to the Onsager matrix from the hydrodynamical expansion of the current. For simplicity we will consider only the third order correction. Following the same steps as in the derivation of the Onsager matrix from the second order, we obtain the contribution

$$
\mathcal{L}_{v, u}=\frac{\ell^{2}}{6}\left(\partial_{\mathrm{q}_{r}(0,0)} \partial_{\mathrm{q}_{j}(0,0)} \partial_{\mathrm{q}_{i}(0,0)}\left\langle\mathrm{j}_{v}(0,0)\right\rangle\right)\left(M_{i j r}^{\mathrm{j}_{u}}-A_{u}^{k} M_{i j r}^{\mathfrak{q}_{k}}\right)+\mathcal{O}\left(\ell^{-1}\right),
$$

with

$$
M_{i j r}^{\mathfrak{o}}=\frac{1}{\ell} \sum_{\chi} \int \mathrm{d} \tau\left\langle\mathfrak{q}_{r}(0, \tau) \mathfrak{q}_{i}(0, \tau) \mathfrak{q}_{j}(0, \tau) \mathfrak{o}(\chi, 0)\right\rangle_{n}^{c} .
$$

Going to the normal mode basis, we obtain

$$
\begin{aligned}
M_{i j r}^{\mathfrak{o}} & =\frac{1}{\ell}\left(R^{-1}\right)_{r}^{r^{\prime}}\left(R^{-1}\right)_{i}^{i^{\prime}}\left(R^{-1}\right)_{j}^{j^{\prime}} \times \\
& \times \sum_{\chi} \int \mathrm{d} \tau \int_{-\pi}^{\pi} \frac{\mathrm{d} k}{2 \pi} \frac{\mathrm{d} k^{\prime}}{2 \pi} \frac{\mathrm{d} k^{\prime \prime}}{2 \pi} e^{-\mathrm{i}\left(k+k^{\prime}+k^{\prime \prime}\right) \chi} e^{-\mathrm{i}\left(\omega_{i^{\prime}}(k)+\omega_{j^{\prime}}\left(k^{\prime}\right)+\omega_{r^{\prime}}\left(k^{\prime \prime}\right)\right) \tau} \times \\
& \times\left\langle\mathfrak{n}_{i^{\prime}}(0,0) \mathfrak{n}_{j^{\prime}}(0,0) \mathfrak{n}_{r^{\prime}}(0,0) \mathfrak{o}(0,0)\right\rangle_{n}^{c} .
\end{aligned}
$$

Summation over $\chi$ and integration over time produces

$$
\begin{aligned}
M_{i j r}^{\mathfrak{o}} & =\frac{1}{\ell}\left(R^{-1}\right)_{r}^{r^{\prime}}\left(R^{-1}\right)_{i}^{i^{\prime}}\left(R^{-1}\right)_{j}^{j^{\prime}} \times \\
& \times \int_{-\pi}^{\pi} \frac{\mathrm{d} k}{2 \pi} \frac{\mathrm{d} k^{\prime}}{2 \pi} \frac{\mathrm{d} k^{\prime \prime}}{2 \pi} 2 \pi \delta\left(k+k^{\prime}+k^{\prime \prime}\right) 2 \pi \delta\left(\left(\omega_{i^{\prime}}(k)+\omega_{j^{\prime}}\left(k^{\prime}\right)+\omega_{r^{\prime}}\left(k^{\prime \prime}\right)\right)\right) \times \\
& \times\left\langle\mathfrak{n}_{i^{\prime}}(0,0) \mathfrak{n}_{j^{\prime}}(0,0) \mathfrak{n}_{r^{\prime}}(0,0) \mathfrak{o}(0,0)\right\rangle_{n}^{c} .
\end{aligned}
$$

Let's assume that $v_{i} \geq v_{j} \geq v_{r}$. Integrating over $k^{\prime \prime}$, we get

$$
\begin{aligned}
M_{i j r}^{\mathfrak{o}} & =\frac{1}{\ell}\left(R^{-1}\right)_{r}^{r^{\prime}}\left(R^{-1}\right)_{i}^{i^{\prime}}\left(R^{-1}\right)_{j}^{j^{\prime}} \times \\
& \times \int_{-\pi}^{\pi} \frac{\mathrm{d} k \mathrm{~d} k^{\prime}}{2 \pi} \delta\left(\left(\omega_{i^{\prime}}(k)+\omega_{j^{\prime}}\left(k^{\prime}\right)+\omega_{r^{\prime}}\left(-k^{\prime}-k\right)\right)\right) \times \\
& \times\left\langle\mathfrak{n}_{i^{\prime}}(0,0) \mathfrak{n}_{j^{\prime}}(0,0) \mathfrak{n}_{r^{\prime}}(0,0) \mathfrak{o}(0,0)\right\rangle_{n}^{c} .
\end{aligned}
$$

Integration over $k$ and $k^{\prime}$ finally yields

$$
M_{i j r}^{\mathfrak{o}}=\frac{1}{\ell}\left(R^{-1}\right)_{r}^{r^{\prime}}\left(R^{-1}\right)_{i}^{i^{\prime}}\left(R^{-1}\right)_{j}^{j^{\prime}} \frac{1}{v_{i}-v_{r}}\left\langle\mathfrak{n}_{i^{\prime}}(0,0) \mathfrak{n}_{j^{\prime}}(0,0) \mathfrak{n}_{r^{\prime}}(0,0) \mathfrak{o}(0,0)\right\rangle_{n}^{c} .
$$

Obviously the contribution vanish in the limit $\ell \rightarrow \infty$, provided that the degeneracies are absent.

\section{References}

[1] J. Sirker, R. G. Pereira and I. Affleck, Conservation laws, integrability, and transport in one-dimensional quantum systems, Phys. Rev. B 83, 035115 (2011), doi:10.1103/PhysRevB.83.035115. 
[2] R. Vasseur and J. E. Moore, Nonequilibrium quantum dynamics and transport: from integrability to many-body localization, Journal of Statistical Mechanics: Theory and Experiment 2016(6), 064010 (2016), doi:10.1088/1742-5468/2016/06/064010.

[3] E. Ilievski, M. Medenjak, T. Prosen and L. Zadnik, Quasilocal charges in integrable lattice systems, Journal of Statistical Mechanics: Theory and Experiment 2016(6), 064008 (2016), doi:10.1088/1742-5468/2016/06/064008.

[4] W. D. Roeck and J. Fröhlich, Diffusion of a massive quantum particle coupled to a quasifree thermal medium, Communications in Mathematical Physics 303(3), 613 (2011), doi:10.1007/s00220-011-1222-0.

[5] C. Karrasch, J. H. Bardarson and J. E. Moore, Reducing the numerical effort of finitetemperature density matrix renormalization group calculations, New Journal of Physics 15(8), 083031 (2013), doi:10.1088/1367-2630/15/8/083031

[6] E. Leviatan, F. Pollmann, J. H. Bardarson, D. A. Huse and E. Altman, Quantum thermalization dynamics with matrix-product states (2017), arXiv:1702.08894.

[7] B. Kloss, Y. B. Lev and D. Reichman, Time-dependent variational principle in matrixproduct state manifolds: Pitfalls and potential, Phys. Rev. B 97, 024307 (2018), doi:10.1103/PhysRevB.97.024307.

[8] S. A. Hartnoll, Theory of universal incoherent metallic transport, Nature Physics 11(1), 54 (2014), doi:10.1038/nphys3174.

[9] A. Lucas and J. Steinberg, Charge diffusion and the butterfly effect in striped holographic matter, Journal of High Energy Physics 2016(10), 143 (2016).

[10] P. Kovtun, D. T. Son and A. O. Starinets, Holography and hydrodynamics: diffusion on stretched horizons, Journal of High Energy Physics 2003(10), 064 (2003), doi: $10.1088 / 1126-6708 / 2003 / 10 / 064$.

[11] S. Grozdanov, D. M. Hofman and N. Iqbal, Generalized global symmetries and dissipative magnetohydrodynamics, Phys. Rev. D 95, 096003 (2017), doi:10.1103/PhysRevD.95.096003.

[12] M. J. Bhaseen, B. Doyon, A. Lucas and K. Schalm, Energy flow in quantum critical systems far from equilibrium, Nat. Phys. 11, 509 EP (2015).

[13] S. Sachdev and J. Ye, Gapless spin-fluid ground state in a random quantum heisenberg magnet, Phys. Rev. Lett. 70, 3339 (1993), doi:10.1103/PhysRevLett.70.3339.

[14] A. A. Patel and S. Sachdev, Critical strange metal from fluctuating gauge fields in a solvable random model, Phys. Rev. B 98, 125134 (2018), doi:10.1103/PhysRevB.98.125134.

[15] A. Nahum, J. Ruhman and D. A. Huse, Dynamics of entanglement and transport in one-dimensional systems with quenched randomness, Phys. Rev. B 98, 035118 (2018), doi:10.1103/PhysRevB.98.035118.

[16] T. Rakovszky, F. Pollmann and C. W. von Keyserlingk, Diffusive hydrodynamics of out-of-time-ordered correlators with charge conservation, Phys. Rev. X 8, 031058 (2018), doi:10.1103/PhysRevX.8.031058. 
[17] J. L. Lebowitz and J. K. Percus, Kinetic equations and density expansions: Exactly solvable one-dimensional system, Phys. Rev. 155(1), 122 (1967), doi: $10.1103 /$ physrev.155.122.

[18] H. Spohn, Hydrodynamical theory for equilibrium time correlation functions of hard rods, Ann. Phys. 141(2), 353 (1982), doi:10.1016/0003-4916(82)90292-5

[19] H. Spohn, Large Scale Dynamics of Interacting Particles, Springer Berlin Heidelberg, doi:10.1007/978-3-642-84371-6 (1991).

[20] J. L. Lebowitz and J. K. Percus, Kinetic Equations and Density Expansions: Exactly Solvable One-Dimensional System, Physical Review 155(1), 122 (1967), doi:10.1103/physrev.155.122.

[21] M. Medenjak, K. Klobas and T. Prosen, Diffusion in deterministic interacting lattice systems, Phys. Rev. Lett. 119, 110603 (2017), doi:10.1103/PhysRevLett.119.110603.

[22] J. De Nardis, D. Bernard and B. Doyon, Hydrodynamic diffusion in integrable systems, Phys. Rev. Lett. 121, 160603 (2018), doi:10.1103/PhysRevLett.121.160603.

[23] S. Gopalakrishnan, D. A. Huse, V. Khemani and R. Vasseur, Hydrodynamics of operator spreading and quasiparticle diffusion in interacting integrable systems, Phys. Rev. B 98, 220303(R) (2018), doi:10.1103/PhysRevB.98.220303.

[24] M. Panfil and J. Pawełczyk, Linearized regime of the generalized hydrodynamics with diffusion (2019), arXiv:1905.06257.

[25] H. Spohn, Interacting and noninteracting integrable systems, Journal of Mathematical Physics 59(9), 091402 (2018).

[26] T. Prosen and D. K. Campbell, Momentum conservation implies anomalous energy transport in 1d classical lattices, Phys. Rev. Lett. 84, 2857 (2000), doi:10.1103/PhysRevLett.84.2857.

[27] N. G. Berloff, M. Brachet and N. P. Proukakis, Modeling quantum fluid dynamics at nonzero temperatures, Proceedings of the National Academy of Sciences 111(Supplement_1), 4675 (2014), doi:10.1073/pnas.1312549111.

[28] M. Kulkarni, D. A. Huse and H. Spohn, Fluctuating hydrodynamics for a discrete grosspitaevskii equation: Mapping onto the kardar-parisi-zhang universality class, Phys. Rev. A 92, 043612 (2015), doi:10.1103/PhysRevA.92.043612.

[29] H. Spohn, Nonlinear fluctuating hydrodynamics for anharmonic chains, Journal of Statistical Physics 154(5), 1191 (2014), doi:10.1007/s10955-014-0933-y.

[30] K. Klobas, M. Medenjak and T. Prosen, Exactly solvable deterministic lattice model of crossover between ballistic and diffusive transport, Journal of Statistical Mechanics: Theory and Experiment 2018(12), 123202 (2018).

[31] J. D. Nardis, D. Bernard and B. Doyon, Diffusion in generalized hydrodynamics and quasiparticle scattering, SciPost Phys. 6, 49 (2019), doi:10.21468/SciPostPhys.6.4.049. 
[32] T. Prosen, Lower bounds on high-temperature diffusion constants from quadratically extensive almost-conserved operators, Phys. Rev. E 89, 012142 (2014), doi:10.1103/PhysRevE.89.012142.

[33] M. Medenjak, C. Karrasch and T. Prosen, Lower bounding diffusion constant by the curvature of drude weight, Phys. Rev. Lett. 119, 080602 (2017), doi:10.1103/PhysRevLett.119.080602.

[34] C. B. Mendl and H. Spohn, Dynamic correlators of fermi-pasta-ulam chains and nonlinear fluctuating hydrodynamics, Phys. Rev. Lett. 111, 230601 (2013), doi:10.1103/PhysRevLett.111.230601.

[35] V. Popkov, A. Schadschneider, J. Schmidt and G. M. Schütz, Fibonacci family of dynamical universality classes, Proceedings of the National Academy of Sciences 112(41), 12645 (2015), doi:10.1073/pnas.1512261112.

[36] M. Kardar, G. Parisi and Y.-C. Zhang, Dynamic Scaling of Growing Interfaces, Phys. Rev. Lett. 56, 889 (1986), doi:10.1103/PhysRevLett.56.889.

[37] R. Kubo, M. Toda and N. Hashitsume, Statistical physics II: nonequilibrium statistical mechanics, vol. 31, Springer Science \& Business Media (2012).

[38] J. M. Deutsch, Eigenstate thermalization hypothesis, Reports on Progress in Physics 81(8), 082001 (2018), doi:10.1088/1361-6633/aac9f1.

[39] L. D'Alessio, Y. Kafri, A. Polkovnikov and M. Rigol, From quantum chaos and eigenstate thermalization to statistical mechanics and thermodynamics, Advances in Physics 65(3), 239 (2016).

[40] B. Doyon, Diffusion and superdiffusion from hydrodynamic projection (2019), 1912. 01551.

[41] X. Zotos, Finite temperature drude weight of the one-dimensional spin- 1/2 heisenberg model, Phys. Rev. Lett. 82, 1764 (1999), doi:10.1103/PhysRevLett.82.1764.

[42] X. Zotos, F. Naef and P. Prelovšek, Transport and conservation laws, Phys. Rev. B 55, 11029 (1997), doi:10.1103/PhysRevB.55.11029.

[43] B. Doyon and H. Spohn, Drude Weight for the Lieb-Liniger Bose Gas, SciPost Phys. 3, 039 (2017), doi:10.21468/SciPostPhys.3.6.039.

[44] H. Spohn, Interacting and noninteracting integrable systems, Journal of Mathematical Physics 59(9), 091402 (2018), doi:10.1063/1.5018624.

[45] E. Ilievski, J. De Nardis, M. Medenjak and T. Prosen, Superdiffusion in one-dimensional quantum lattice models, Phys. Rev. Lett. 121, 230602 (2018), doi:10.1103/PhysRevLett.121.230602.

[46] B. Bertini, M. Collura, J. De Nardis and M. Fagotti, Transport in Out-of-Equilibrium XXZ Chains: Exact Profiles of Charges and Currents, Phys. Rev. Lett. 117, 207201 (2016), doi:10.1103/PhysRevLett.117.207201. 
[47] B. Doyon and T. Yoshimura, A note on generalized hydrodynamics: inhomogeneous fields and other concepts, SciPost Phys. 2, 014 (2017), doi:10.21468/SciPostPhys.2.2.014.

[48] O. A. Castro-Alvaredo, B. Doyon and T. Yoshimura, Emergent hydrodynamics in integrable quantum systems out of equilibrium, Phys. Rev. X 6, 041065 (2016), doi:10.1103/PhysRevX.6.041065.

[49] E. Ilievski and J. De Nardis, Microscopic origin of ideal conductivity in integrable quantum models, Phys. Rev. Lett. 119, 020602 (2017), doi:10.1103/PhysRevLett.119.020602.

[50] B. Doyon, T. Yoshimura and J.-S. Caux, Soliton gases and generalized hydrodynamics, Phys. Rev. Lett. 120, 045301 (2018), doi:10.1103/PhysRevLett.120.045301.

[51] V. B. Bulchandani, R. Vasseur, C. Karrasch and J. E. Moore, Bethe-boltzmann hydrodynamics and spin transport in the xxz chain, Phys. Rev. B 97, 045407 (2018), doi:10.1103/PhysRevB.97.045407.

[52] E. Ilievski, E. Quinn and J.-S. Caux, From interacting particles to equilibrium statistical ensembles, Phys. Rev. B 95, 115128 (2017), doi:10.1103/PhysRevB.95.115128.

[53] A. J. Friedman, S. Gopalakrishnan and R. Vasseur, Integrable manybody quantum floquet-thouless pumps, Phys. Rev. Lett. 123, 170603 (2019), doi:10.1103/PhysRevLett.123.170603.

[54] J. D. Nardis, M. Medenjak, C. Karrasch and E. Ilievski, Anomalous spin diffusion in one-dimensional antiferromagnets (2019), arXiv:1903.07598.

[55] L. Bertini and G. Giacomin, Stochastic burgers and KPZ equations from particle systems, Communications in Mathematical Physics 183(3), 571 (1997), doi: $10.1007 / \mathrm{s} 002200050044$.

[56] M. Ljubotina, M. Žnidarič and T. Prosen, Kardar-parisi-zhang physics in the quantum heisenberg magnet (2019), arXiv:1903.01329.

[57] A. Das, M. Kulkarni, H. Spohn and A. Dhar, Kardar-parisi-zhang scaling for an integrable lattice landau-lifshitz spin chain, Phys. Rev. E 100, 042116 (2019), doi:10.1103/PhysRevE.100.042116.

[58] M. Dupont and J. E. Moore, Universal spin dynamics in infinite-temperature onedimensional quantum magnets (2019), arXiv:1907.12115.

[59] F. Weiner, P. Schmitteckert, S. Bera and F. Evers, High-temperature spin dynamics in the heisenberg chain: Magnon propagation and emerging kpz-scaling in the zero magnetization limit (2019), arXiv:1908.11432.

[60] M. Bauer, D. Bernard and T. Jin, Stochastic dissipative quantum spin chains (I) : Quantum fluctuating discrete hydrodynamics, SciPost Phys. 3, 033 (2017), doi: $10.21468 /$ SciPostPhys.3.5.033.

[61] S. Gopalakrishnan and R. Vasseur, Kinetic theory of spin diffusion and superdiffusion in xxz spin chains, Phys. Rev. Lett. 122, 127202 (2019), doi:10.1103/PhysRevLett.122.127202. 
[62] V. B. Bulchandani, Kardar-parisi-zhang universality from soft gauge modes (2019), arXiv: 1910.08266.

[63] L. Piroli, J. De Nardis, M. Collura, B. Bertini and M. Fagotti, Transport in out-ofequilibrium XXZ chains: Nonballistic behavior and correlation functions, Phys. Rev. B 96, 115124 (2017), doi:10.1103/PhysRevB.96.115124.

[64] J. G. Kirkwood, The statistical mechanical theory of transport processes i. general theory, The Journal of Chemical Physics 14(3), 180 (1946), doi:10.1063/1.1724117.

[65] M. Born and H. Green, A general kinetic theory of liquids i. the molecular distribution functions, Proceedings of the Royal Society of London. Series A. Mathematical and Physical Sciences 188(1012), 10 (1946).

[66] G. Policastro, D. T. Son and A. O. Starinets, Shear viscosity of strongly coupled $n=4$ supersymmetric yang-mills plasma, Phys. Rev. Lett. 87, 081601 (2001), doi:10.1103/PhysRevLett.87.081601. 